\title{
PERTURBATION THEORY FOR HAMILTONIAN MATRICES AND THE DISTANCE TO BOUNDED-REALNESS ${ }^{*}$
}

\author{
R. $\mathrm{ALAM}^{\dagger}, \mathrm{S} . \mathrm{BORA}^{\dagger}, \mathrm{M} . \mathrm{KAROW} W^{\ddagger}, \mathrm{V} \cdot \mathrm{MEHRMANN}^{\ddagger}$, AND J. MORO
}

\begin{abstract}
Motivated by the analysis of passive control systems, we undertake a detailed perturbation analysis of Hamiltonian matrices that have eigenvalues on the imaginary axis. We construct minimal Hamiltonian perturbations that move and coalesce eigenvalues of opposite sign characteristic to form multiple eigenvalues with mixed sign characteristics, which are then moved from the imaginary axis to specific locations in the complex plane by small Hamiltonian perturbations. We also present a numerical method to compute upper bounds for the minimal perturbations that move all eigenvalues of a given Hamiltonian matrix outside a vertical strip along the imaginary axis.
\end{abstract}

Key words. Hamiltonian matrix, Hamiltonian eigenvalue problem, perturbation theory, passive system, bounded-realness, purely imaginary eigenvalues, sign characteristic, Hamiltonian pseudospectra, structured mapping problem, distance to bounded-realness

AMS subject classifications. 93B $36,93 \mathrm{~B} 40,49 \mathrm{~N} 35,65 \mathrm{~F} 15,93 \mathrm{~B} 52,93 \mathrm{C} 05$

DOI. $10.1137 / 10079464 \mathrm{X}$

1. Introduction. In this paper we discuss the perturbation theory for eigenvalues of Hamiltonian matrices and the explicit construction of small perturbations that move eigenvalues from the imaginary axis. With $\mathbb{F}^{k, \ell}$ denoting the vector space of real $(\mathbb{F}=\mathbb{R})$ or complex $(\mathbb{F}=\mathbb{C}) k \times \ell$ matrices, a matrix $\mathcal{H} \in \mathbb{F}^{2 n, 2 n}$ is called Hamiltonian if $(\mathcal{H} \mathcal{J})^{\star}=\mathcal{H} \mathcal{J}$, where $\mathcal{J}=\left[\begin{array}{cc}0 & I_{n} \\ -I_{n} & 0\end{array}\right]$ and $I_{n}$ is the $n \times n$ identity matrix (we suppress the subscript $n$ if the dimension is clear from the context). In order to simplify the presentation and to treat the real and the complex cases together, we use ${ }^{\star}$ to denote ${ }^{T}$ in the real case and ${ }^{*}$ in the complex case.

1.1. The distance to bounded-realness. It is well-known [22], [26] that the spectrum of Hamiltonian matrices is symmetric with respect to the imaginary axis; i.e., eigenvalues occur in pairs $(\lambda,-\bar{\lambda})$ in the complex case or quadruples $(\lambda,-\lambda, \bar{\lambda},-\bar{\lambda})$ in the real case. This eigenvalue symmetry degenerates if there are eigenvalues on the imaginary axis. The existence of purely imaginary eigenvalues typically leads to difficulties for numerical methods in control [7], [26]. If purely imaginary eigenvalues occur, then in some applications (see, e.g., section 1.2) one perturbs the Hamiltonian matrix in such a way that the eigenvalues are moved away from the imaginary axis. We formulate this as our first problem.

Problem A. Given a Hamiltonian matrix $\mathcal{H}$ that has purely imaginary eigenvalues, determine (in some norm to be specified) the smallest Hamiltonian perturbation $\Delta \mathcal{H}$ such that for the resulting perturbed matrix $\mathcal{H}+\Delta \mathcal{H}$ an arbitrary small Hamiltonian

*Received by the editors May 10, 2010; accepted for publication (in revised form) by R.-C. Li April 12, 2011; published electronically June 27, 2011. This research was supported by Deutsche Forschungsgemeinschaft, via the DFG Research Center Matheon in Berlin.

http://www.siam.org/journals/simax/32-2/79464.html

'Department of Mathematics, IIT Guwahati, Assam, India (rafik@iitg.ernet.in, shbora@iitg.ernet.in).

Institut für Mathematik, Ma 4-5, TU Berlin, Straße des 17. Juni 136, D-10623 Berlin, FRG (karow@math .tu-berlin.de, mehrmann@math.tu-berlin.de).

${ }^{\S}$ Departamento de Matemáticas, Universidad Carlos III de Madrid, Avda. Universidad 30, 28911-Leganés, Spain (jmoro@math.uc3m.es). This author's research was partially supported by the Spanish Ministerio de Ciencia y Tecnología under grant MTM2006-05361.

Copyright ( $)$ by SIAM. Unauthorized reproduction of this article is prohibited. 
perturbation will generically move all the eigenvalues off the imaginary axis. (By "generically" it is meant that those small Hamiltonian perturbations which do not move the imaginary eigenvalues away from the axis lie in a subset of zero measure within the set of Hamiltonian matrices.)

Since checking the existence of purely imaginary eigenvalues of a Hamiltonian matrix is used in the context of the bounded real lemma [4], we call this distance the distance to bounded-realness. The converse of this problem of determining the smallest Hamiltonian perturbation of a Hamiltonian matrix so that all eigenvalues of the resulting perturbed matrix are purely imaginary (i.e., the distance to nonbounded-realness) has recently been studied on the basis of so-called $\mu$-values and spectral value sets in [18].

While the distance to bounded-realness is an important quantity that has to be determined in order to characterize whether it is possible to find a perturbation that moves all eigenvalues off the imaginary axis, in applications (see, e.g., section 1.2) often a modified question is more important.

Problem B. Given a Hamiltonian matrix $\mathcal{H}$ that has purely imaginary eigenvalues, determine (in some norm to be specified) the smallest Hamiltonian perturbation $\Delta \mathcal{H}$ such that the resulting perturbed matrix $\mathcal{H}+\Delta \mathcal{H}$ has all eigenvalues robustly bounded away from the imaginary axis; i.e., all eigenvalues of $\mathcal{H}+\Delta \mathcal{H}$ lie outside an open vertical strip $S_{\tau}=\{z \in \mathbb{C} \mid-\tau<\mathfrak{R} z<\tau\}(\tau \geq 0)$ along the imaginary axis.

If a numerically backward stable method is used (and we will propose such a method), then we just have to choose the width of the strip so that perturbations on the order of the round-off errors cannot move eigenvalues on the imaginary axis again. We will discuss such choices below.

In this paper we discuss numerical procedures for the solution of both Problems A and $\mathrm{B}$. We mention that determination of minimal perturbations is in general a difficult nonconvex optimization problem; see [10]. Instead, we construct suboptimal perturbations and hence obtain upper bounds for the smallest perturbations.

1.2. Passivation. The main motivation for studying the perturbation problems that we have discussed in the previous subsection is the following. Consider a linear time-invariant control system

$$
\begin{aligned}
& \dot{x}=A x+B u, \quad x(0)=0, \\
& y=C x+D u,
\end{aligned}
$$

with matrices $A \in \mathbb{F}^{n, n}, B \in \mathbb{F}^{n, m}, C \in \mathbb{F}^{p, n}$, and $D \in \mathbb{F}^{p, m}$. Here $u$ is the input, $x$ the state, and $y$ the output.

Suppose that the homogeneous system is asymptotically stable; i.e., all eigenvalues of $A$ are in the open left half complex plane and that $D$ is square and nonsingular. Then (see, e.g., [4]) the system is called passive if there exists a nonnegative scalar valued function $\Theta$ such that the dissipation inequality

$$
\Theta\left(x\left(t_{1}\right)\right)-\Theta\left(x\left(t_{0}\right)\right) \leq \int_{t_{0}}^{t_{1}}\left(u^{\star} y+y^{\star} u\right) \mathrm{d} t
$$

holds for all $t_{1} \geq t_{0}$; i.e., the system absorbs supply energy.

In real world applications the system model (1.1) is typically subject to several approximations. Often the real physical problem (e.g., the determination of the electric or magnetic field associated with an electronic device) is infinite dimensional and is

Copyright (c) by SIAM. Unauthorized reproduction of this article is prohibited. 
approximated by a finite element or finite difference model [17], or the system is nonlinear and the linear model is obtained by a linearization. The system may also be obtained by a realization or system identification [8], [16], [35], or it may be the result of a model reduction procedure [4].

If one uses an approximated model, then it is in general not clear that the property of passivity will be preserved, and typically it is not; i.e., the approximation process makes the passive system nonpassive. Since passivity is an important physical property (a passive system does not generate energy), one then approximates the nonpassive system by a (hopefully) nearby passive system by introducing small (minimal) perturbations of $A$, $B, C, D$; see [8], [10], [15], [35], [36].

Typically, one has an estimate or even a bound for the approximation error in the original system approximation, and then one tries to keep the perturbations within these bounds. So from the application point of view it may not be necessary to really determine the minimal perturbation; a perturbation that stays within the range of the already committed approximation errors is sufficient. But from a system theoretical point of view, it is also interesting to find a value or a bound for the smallest perturbation that makes a nonpassive system passive. In general it is an open problem to determine this minimal perturbation explicitly. Instead one uses optimization methods [8], [10], [11] or ad hoc perturbation methods [14], [15], [35], [34]; see also [36] for a recent improvement of the method in [15].

The converse problem of computing the smallest perturbation that makes a passive system nonpassive has recently been studied in [29], again using optimization techniques.

At first sight the passivation problem does not seem to be related to the perturbation problem for Hamiltonian matrices. However, it is well-known [4], [15] that one can check whether an asymptotically stable system is passive by checking whether the Hamiltonian matrix

$$
\mathcal{H}=\left[\begin{array}{cc}
F & G \\
H & -F^{\star}
\end{array}\right]:=\left[\begin{array}{cc}
A-B R^{-1} C & -B R^{-1} B^{\star} \\
-C^{\star} R^{-1} C & -\left(A-B R^{-1} C\right)^{\star}
\end{array}\right]
$$

has no purely imaginary eigenvalues, where we have set $R=D+D^{\star}$. Thus one can use the distance to bounded-realness (i.e., perturbations that solve Problems A and B) to construct perturbations that make the system passive. This topic will be discussed in a forthcoming work.

The paper is organized as follows: In section 2 we introduce the notation and briefly present some preliminary results. The perturbation theory for eigenvalues, in particular purely imaginary eigenvalues of Hamiltonian matrices, is reviewed in section 3 . Hamiltonian perturbations moving purely imaginary eigenvalues of a Hamiltonian matrix to specific points in the complex plane are discussed in section 4 . The minimal perturbations or bounds of minimal perturbations are discussed in section 5 . A numerical method to compute approximate solutions of Problems A and B for the spectral norm $\|\cdot\|_{2}$ is discussed in section 6 .

2. Preliminaries. By $\mathbb{C}_{+}$and $\mathbb{C}_{-}$, respectively, we denote the positive right half and negative left half complex plane. For $X \in \mathbb{F}^{n, m}$ of full column rank, we denote by $X^{+}:=\left(X^{\star} X\right)^{-1} X^{\star}$ the Moore-Penrose inverse of $X$; see, e.g., [13]. For $A \in \mathbb{F}^{n, n}$, the spectrum is denoted by $\Lambda(A)$. A subspace $\mathcal{X} \subseteq \mathbb{F}^{n}$ is said to be $A$-invariant if $A x \in \mathcal{X}$ for any $x \in \mathcal{X}$. In this case we denote by $\Lambda(A \mid \mathcal{X})$ the spectrum of the restriction of the linear operator $A$ to the subspace $\mathcal{X}$. Let $X \in \mathbb{F}^{n, d}$ be a full column rank matrix 
such that $\mathcal{X}=\operatorname{range}(X)$. Then $\mathcal{X}$ is $A$-invariant if $A X=X R$ for some $R \in \mathbb{F}^{d, d}$, and then we have $\Lambda(A \mid \mathcal{X})=\Lambda(R)$.

It is well-known [28], [31], [32] that the Hermitian form

$$
(x, y) \mapsto i x^{\star} \mathcal{J} y, \quad x, y \in \mathbb{F}^{2 n},
$$

plays an important role in the perturbation theory of Hamiltonian eigenvalues. If $x^{\star} \mathcal{J} y=0$, then $x$ and $y$ are said to be $\mathcal{J}$-orthogonal. Subspaces $\mathcal{X}, \mathcal{Y} \subseteq \mathbb{F}^{2 n}$ are said to be $\mathcal{J}$-orthogonal if $x^{\star} \mathcal{J} y=0$ for all $x \in \mathcal{X}, y \in \mathcal{Y}$. A subspace $\mathcal{X} \subseteq \mathbb{F}^{2 n}$ is said to be $\mathcal{J}$-neutral if $x^{\star} \mathcal{J} x=0$ for all $x \in \mathcal{X}$. $\mathcal{X}$ is said to be $\mathcal{J}$-nondegenerate if for any $x \in$ $\mathcal{X} \backslash\{0\}$ there exists $y \in \mathcal{X}$ such that $x^{\star} \mathcal{J} y \neq 0$.

Nondegenerate invariant subspaces for Hamiltonian matrices are characterized by the following theorem, where for a set of complex numbers $\Xi=\left\{\xi_{1}, \ldots, \xi_{k}\right\}$ we denote by $\bar{\Xi}$ the set of conjugates of the elements of $\Xi$.

Theorem 2.1 (see [12]). Let $\mathcal{X}_{1}$ and $\mathcal{X}_{2}$ be invariant subspaces of the Hamiltonian matrix $\mathcal{H} \in \mathbb{F}^{2 n, 2 n}$. Suppose that $\Lambda\left(\mathcal{H} \mid \mathcal{X}_{1}\right) \cap\left(-\overline{\Lambda\left(\mathcal{H} \mid \mathcal{X}_{2}\right)}\right)=\varnothing$. Then $x_{1}^{\star} \mathcal{J} x_{2}=0$ for all $x_{1} \in \mathcal{X}_{1}, x_{2} \in \mathcal{X}_{2}$.

Suppose, additionally, that $\mathcal{X}_{1} \oplus \mathcal{X}_{2}=\mathbb{F}^{2 n}$. Then $\mathcal{X}_{1}$ and $\mathcal{X}_{2}$ are $\mathcal{J}$-nondegenerate.

Proof. Let $X_{k} \in \mathbb{F}^{2 n, p_{k}}$ be a matrix whose columns form a basis of $\mathcal{X}_{k}, k=1,2$. Then $\mathcal{H} X_{k}=X_{k} R_{k}$, and the matrix $R_{k} \in \mathbb{F}^{p_{k}, p_{k}}$ satisfies $\Lambda\left(R_{k}\right)=\Lambda\left(\mathcal{H} \mid \mathcal{X}_{k}\right)$. Consider the $S y l$ vester operator $\mathcal{S}(Z)=R_{1}^{\star} Z+Z R_{2}, Z \in \mathbb{F}^{p_{1}, p_{2}}$. We have

$$
\begin{aligned}
\mathcal{S}\left(X_{1}^{\star} \mathcal{J} X_{2}\right) & =R_{1}^{\star} X_{1}^{\star} \mathcal{J} X_{2}+X_{1}^{\star} \mathcal{J} X_{2} R_{2} \\
& =-\left(\mathcal{J} X_{1} R_{1}\right)^{\star} X_{2}+X_{1}^{\star}\left(\mathcal{J} X_{2} R_{2}\right) \\
& =-\left(\mathcal{J} \mathcal{H} X_{1}\right)^{\star} X_{2}+X_{1}^{\star}\left(\mathcal{J H} X_{2}\right) \\
& =-X_{1}^{\star}(\mathcal{J H})^{\star} X_{2}+X_{1}^{\star}(\mathcal{J H}) X_{2} \\
& =0 .
\end{aligned}
$$

Furthermore, by assumption $0 \notin \Lambda\left(R_{1}^{\star}\right)+\Lambda\left(R_{2}\right)$ and thus the Sylvester operator $\mathcal{S}$ is nonsingular [23]. Hence we have $X_{1}^{\star} \mathcal{J} X_{2}=0$, and this completes the proof of the first claim.

For the second part, suppose that $\mathcal{X}_{1} \oplus \mathcal{X}_{2}=\mathbb{F}^{2 n}$ and that $\mathcal{X}_{1}$ is degenerate. Then there exists $x_{1} \in \mathcal{X}_{1} \backslash\{0\}$ such that $x_{1}^{\star} \mathcal{J} x=0$ for all $x \in \mathcal{X}_{1}$. However, we also have $x_{1}^{\star} \mathcal{J} x=0$ for all $x \in \mathcal{X}_{2}$. This yields $x_{1}^{\star} \mathcal{J}=0$, contradicting the nonsingularity of $\mathcal{J}$.

We immediately have the following corollary; see, e.g., [12].

Corollary 2.2. Let $\mathcal{H} \in \mathbb{F}^{2 n, 2 n}$ be Hamiltonian. Let $i \alpha_{1}, \ldots, i \alpha_{p} \in i \mathbb{R}$ be the purely imaginary eigenvalues of $\mathcal{H}$, and let $\lambda_{1}, \ldots, \lambda_{q} \in \mathbb{C}$ be the eigenvalues of $\mathcal{H}$ with negative real part. Then the $\mathcal{H}$-invariant subspaces $\operatorname{ker}\left(\mathcal{H}-i \alpha_{k} I\right)^{2 n}$ and $\operatorname{ker}\left(\mathcal{H}-\lambda_{j} I\right)^{2 n} \bigoplus$ $\operatorname{ker}\left(\mathcal{H}+\overline{\lambda_{j}} I\right)^{2 n}$ are pairwise $\mathcal{J}$-orthogonal. All these subspaces are $\mathcal{J}$-nondegenerate. The subspaces

$$
\begin{aligned}
& \mathcal{X}_{-}(\mathcal{H}):=\bigoplus_{j=1}^{q} \operatorname{ker}\left(\mathcal{H}-\lambda_{j} I\right)^{2 n}, \\
& \mathcal{X}_{+}(\mathcal{H}):=\bigoplus_{j=1}^{q} \operatorname{ker}\left(\mathcal{H}+\bar{\lambda}_{j} I\right)^{2 n}
\end{aligned}
$$

are $\mathcal{J}$-neutral. 
There are several viewpoints that can be taken to perform the perturbation analysis for Hamiltonian matrices. We will mostly work with the quadratic form (2.1). Another approach would be to use the normal and condensed forms for Hamiltonian matrices under symplectic or unitary symplectic transformations, respectively [24], [26]. Recall that a matrix $\mathcal{S}$ is called symplectic if $\mathcal{S}^{\star} \mathcal{J S}=\mathcal{J}$ and it is called unitary (orthogonal in the real case) symplectic if $\mathcal{S}$ is symplectic and $\mathcal{S}^{\star} \mathcal{S}=I$.

The normal form under symplectic transformations forms the basis for the computation of eigenvalues, eigenvectors, and invariant subspaces of Hamiltonian matrices. But since the group of symplectic matrices is not compact, to obtain backward stable numerical methods it is important to use unitary (orthogonal) symplectic matrices for the transformations. In this case, in general, we cannot get the complete spectral information but only a condensed form - the (partial) Hamiltonian Schur form.

Lemma 2.3 (see [25], [26]). Given a Hamiltonian matrix $\mathcal{H} \in \mathbb{F}^{2 n, 2 n}$, there exists a unitary symplectic (real orthogonal symplectic if $\mathbb{F}=\mathbb{R}$ ) matrix $Q \in \mathbb{F}^{2 n, 2 n}$ such that

$$
\mathcal{T}=Q^{\star} \mathcal{H} Q=\left[\begin{array}{cc|rc}
F_{11} & F_{12} & G_{11} & G_{12} \\
0 & F_{22} & G_{21} & G_{22} \\
\hline 0 & 0 & -F_{11}^{\star} & 0 \\
0 & H_{22} & -F_{12}^{\star} & -F_{22}^{\star}
\end{array}\right] \text {, }
$$

where $F_{11}$ is upper triangular (quasi-upper triangular in the real case) and has only eigenvalues in the open left half-plane, while the submatrix

$$
\left[\begin{array}{c|c}
F_{22} & G_{22} \\
\hline H_{22} & -F_{22}^{\star}
\end{array}\right]
$$

has only purely imaginary eigenvalues. If there are no purely imaginary eigenvalues, then this latter block is void, and this becomes a Hamiltonian Schur form.

Under further conditions (see [9], [24], [25]) a Hamiltonian Schur form also exists if purely imaginary eigenvalues occur.

It is worth mentioning that if 0 is an eigenvalue, then it is treated differently for real and nonreal Hamiltonian matrices. Indeed, for nonreal Hamiltonian matrices 0 is considered to be purely imaginary. In contrast, for real Hamiltonian matrices the eigenvalue 0 plays a special role, and in some of the literature (see, e.g., [12]) it is even considered to be not on the imaginary axis. For us, however, 0 will be treated as purely imaginary.

We now discuss the perturbation theory for purely imaginary eigenvalues of Hamiltonian matrices.

3. Perturbation theory for Hamiltonian matrices. In this section we discuss perturbation results for Hamiltonian matrices. In particular, we analyze how purely imaginary eigenvalues of Hamiltonian matrices behave under Hamiltonian perturbations, and then we characterize when small perturbations allow one to move purely imaginary eigenvalues away from the imaginary axis; see also [21], [28], [30], [31], [32]. To be more precise, given a Hamiltonian matrix $\mathcal{H} \in \mathbb{F}^{2 n, 2 n}$ with a purely imaginary eigenvalue $i \alpha$, our primary aim is to determine a minimal Hamiltonian perturbation $\Delta \mathcal{H}$ such that $i \alpha$ moves away from the imaginary axis to some specified location in the complex plane when $\mathcal{H}$ is perturbed to $\mathcal{H}+\Delta \mathcal{H}$. By minimal perturbation we mean that $\Delta \mathcal{H}$ has the smallest norm, either in the Frobenius or in the spectral norm.

Copyright ( by SIAM. Unauthorized reproduction of this article is prohibited. 
It is well-known that the spectral perturbation theory for Hamiltonian matrices [28], [30], [31], in particular for the purely imaginary eigenvalues, is substantially different from the well-known classical perturbation theory for eigenvalues and eigenvectors of unstructured matrices; see, e.g., [37].

Let $\mathcal{H} \in \mathbb{F}^{2 n, 2 n}$ be Hamiltonian, and suppose that $i \alpha$ is a purely imaginary eigenvalue of $\mathcal{H}$. Let $X$ be a full column rank matrix such that the columns of $X$ span the right invariant subspace $\operatorname{ker}(\mathcal{H}-i \alpha I)^{2 n}$ associated with $i \alpha$ so that

$$
\mathcal{H} X=X R \quad \text { and } \quad \Lambda(R)=\{i \alpha\}
$$

for some square matrix $R$. By using the fact that $\mathcal{H}$ is Hamiltonian, we also have

$$
X^{\star} \mathcal{J H}=-R^{\star} X^{\star} \mathcal{J} .
$$

Since $\Lambda\left(-R^{\star}\right)=\{i \alpha\}$, it follows that the columns of the full column rank matrix $\mathcal{J}^{\star} X$ span the left invariant subspace associated with $i \alpha$. Hence $\left(J^{\star} X\right)^{\star} X=X^{\star} \mathcal{J} X$ is nonsingular and the matrix

$$
Z=i X^{\star} \mathcal{J} X
$$

associated with the Hermitian form (2.1) is nonsingular. This leads to the following perturbation result for the spectral norm $\|\cdot\|_{2}$.

Theorem 3.1 (see [28]). Consider a Hamiltonian matrix $\mathcal{H} \in \mathbb{F}^{2 n, 2 n}$ with a purely

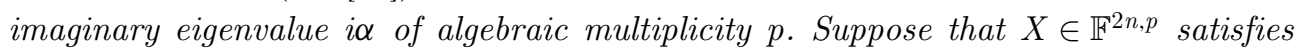
$\operatorname{rank} X=p$ and (3.1), and that $Z$ as defined in (3.3) is congruent to $\left[\begin{array}{cc}I_{\pi} & 0 \\ 0 & I_{\mu}\end{array}\right]$ (with $\pi+\mu=p)$.

If $\Delta \mathcal{H}$ is Hamiltonian and $\|\Delta \mathcal{H}\|_{2}$ is sufficiently small, then $\mathcal{H}+\Delta \mathcal{H}$ has $p$ eigenvalues $\lambda_{1}, \ldots, \lambda_{p}$ (counting multiplicity) in the neighborhood of $i \alpha$, among which at least $|\pi-\mu|$ eigenvalues are purely imaginary. In particular, we have the following cases.

1. If $Z$ is definite (i.e., either $\pi=0$ or $\mu=0$ ), then all $\lambda_{1}, \ldots, \lambda_{p}$ are purely imaginary with equal algebraic and geometric multiplicity, and satisfy

$$
\lambda_{j}=i\left(\alpha+\delta_{j}\right)+O\left(\|\Delta \mathcal{H}\|_{2}^{2}\right)
$$

where $\delta_{1}, \ldots, \delta_{p}$ are the real eigenvalues of the pencil $\lambda Z-X^{\star}(\mathcal{J} \Delta \mathcal{H}) X$.

2. If there exists a Jordan block associated with $i \alpha$ of size larger than 2 , then generically for a given $\Delta \mathcal{H}$ some eigenvalues among $\lambda_{1}, \ldots, \lambda_{p}$ will no longer be purely imaginary.

If there exists a Jordan block associated with i $\alpha$ of size 2 , then for any $\epsilon>0$, there always exists a Hamiltonian perturbation matrix $\Delta \mathcal{H}$ with $\|\Delta \mathcal{H}\|_{2}=\epsilon$ such that some eigenvalues among $\lambda_{1}, \ldots, \lambda_{p}$ will have nonzero real part.

3. If $i \alpha$ has equal algebraic and geometric multiplicity and $Z$ is indefinite, then for any $\epsilon>0$, there always exists a Hamiltonian perturbation matrix $\Delta \mathcal{H}$ with $\|\Delta \mathcal{H}\|_{2}=\epsilon$ such that some eigenvalues among $\lambda_{1}, \ldots, \lambda_{p}$ will have nonzero real part.

We now revisit the perturbation results in Theorem 3.1 and present them in a form that we can directly use in the construction of small perturbations. In what follows, we show that an imaginary eigenvalue of $\mathcal{H}$ can be moved off the imaginary axis by an arbitrary small Hamiltonian perturbation if and only if $\mathcal{H}$ has a $\mathcal{J}$-neutral eigenvector

Copyright ( $)$ by SIAM. Unauthorized reproduction of this article is prohibited. 
corresponding to the imaginary eigenvalue. We then describe how to construct such a Hamiltonian perturbation.

Suppose that we wish to construct a Hamiltonian perturbation matrix $\mathcal{E}$ of smallest norm such that an eigenvalue of $\mathcal{H}$ moves to $\mu$ when $\mathcal{H}$ is perturbed to $\mathcal{H}+\mathcal{E}$. For such a perturbation then there exists a vector $u$ such that $(\mathcal{H}+\mathcal{E}) u=\mu u$. This means that $\mathcal{E} u=\mu u-\mathcal{H} u=r$. Thus the resulting $\mathcal{E}$ is a solution of the following structured mapping problem (see [1], [2]): Given $x, b \in \mathbb{F}^{2 n}$ find a Hamiltonian matrix $\mathcal{G}$ of smallest norm $\|\mathcal{G}\|$ such that $\mathcal{G} x=b$. Here $\|\cdot\|$ is either the spectral norm or the Frobenius norm.

To solve this problem in a more general framework, for $X \in \mathbb{F}^{2 n, p}$ and $B \in \mathbb{F}^{2 n, p}$, we introduce

$$
\eta(X, B):=\inf \left\{\|\mathcal{H}\|: \mathcal{H} \in \mathbb{F}^{2 n, 2 n},(\mathcal{J H})^{\star}=\mathcal{J} \mathcal{H} \text { and } \mathcal{H} X=B\right\}
$$

denoting $\eta(X, B)$ by $\eta_{F}(X, B)$ for the Frobenius norm and by $\eta_{2}(X, B)$ for the spectral norm. The following result, taken from [1], [2], provides a complete solution of the Hamiltonian structured mapping problem.

Theorem 3.2 (see [1], [2]).

(a)

1. Let $x \in \mathbb{F}^{2 n}$ and $b \in \mathbb{F}^{2 n}$. Then there exists a Hamiltonian matrix $\mathcal{H} \in \mathbb{F}^{2 n, 2 n}$ such that $\mathcal{H} x=b$ if and only if $x^{\star} \mathcal{J} b \in \mathbb{R}$.

2. If $x^{\star} \mathcal{J} b$ is real, then

$$
\begin{aligned}
\eta_{F}(x, b) & =\sqrt{2\|b\|_{2}^{2} /\|x\|_{2}^{2}-\left|x^{\star} \mathcal{J} b\right|^{2} /\|x\|_{2}^{4}}, \\
\eta_{2}(x, b) & =\|b\|_{2} /\|x\|_{2} .
\end{aligned}
$$

Furthermore, the matrix

$$
\mathcal{G}(x, b):=\frac{b x^{\star}+\mathcal{J} x b^{\star} \mathcal{J}}{\|x\|_{2}^{2}}+\frac{\left(x^{\star} \mathcal{J} b\right) \mathcal{J} x x^{\star}}{\|x\|_{2}^{4}}
$$

is the unique Hamiltonian matrix satisfying $\mathcal{G}(x, b) x=b$ and $\|\mathcal{G}(x, b)\|_{F}=$ $\eta_{F}(x, b)$.

3. If $\|x\|_{2}\|b\|_{2} \neq\left|x^{\star} \mathcal{J} b\right|$, then form the Hamiltonian matrix

$$
\mathcal{F}(x, b):=\mathcal{G}(x, b)-\frac{x^{\star} \mathcal{J} b}{\|b\|_{2}^{2}\|x\|_{2}^{2}-\left|x^{\star} \mathcal{J} b\right|^{2}} \mathcal{J}\left(I-\frac{x x^{\star}}{x^{\star} x}\right) \mathcal{J} b b^{\star} \mathcal{J}\left(I-\frac{x x^{\star}}{x^{\star} x}\right) ;
$$

otherwise, set $\mathcal{F}(x, b):=\mathcal{G}(x, b)$. Then $\mathcal{F}(x, b) x=b$ and $\|\mathcal{F}(x, b)\|_{2}=$ (b) $\eta_{2}(x, b)$.

1. Let $B \in \mathbb{F}^{2 n, p}$ and $X \in \mathbb{F}^{2 n, p}$. Suppose that $\operatorname{rank} X=p$. Then there exists a Hamiltonian matrix $\mathcal{H} \in \mathbb{F}^{2 n, 2 n}$ such that $\mathcal{H} X=B$ if and only if $X^{\star} \mathcal{J} B$ is Hermitian.

2. If $X^{\star} \mathcal{J} B$ is Hermitian, then

$$
\begin{aligned}
\eta_{2}(X, B) & =\left\|B\left(X^{\star} X\right)^{-1 / 2}\right\|_{2}, \\
\eta_{F}(X, B) & =\sqrt{2\left\|B\left(X^{\star} X\right)^{-1 / 2}\right\|_{F}^{2}-\left\|\left(X^{\star} X\right)^{-1 / 2} X^{\star} \mathcal{J} B\left(X^{\star} X\right)^{-1 / 2}\right\|_{F}^{2}} .
\end{aligned}
$$

3. Let $X^{+}$denote the Moore-Penrose inverse of $X$. Then

Copyright () by SIAM. Unauthorized reproduction of this article is prohibited. 


$$
\mathcal{G}(X, B):=B X^{+}+\mathcal{J}\left(X^{+}\right)^{\star} B^{\star} \mathcal{J}+\mathcal{J} X X^{+} \mathcal{J} B X^{+}
$$

is the unique Hamiltonian matrix satisfying $\mathcal{G}(X, B) X=B$ and $\|\mathcal{G}(X, B)\|_{F}=\eta_{F}(X, B)$.

4. Set $Z:=\left(X^{\star} X\right)^{-1 / 2} X^{\star} \mathcal{J} B\left(X^{\star} X\right)^{-1 / 2}$ and $\rho:=\eta_{2}(X, B)$. If $\rho^{2} I-Z^{2}$ is nonsingular, then consider the Hamiltonian matrix

$$
\mathcal{F}(X, B):=\mathcal{G}(X, B)+\mathcal{J}\left(I-X X^{+}\right) K Z K^{\star}\left(I-X X^{+}\right),
$$

where $K:=\mathcal{J} B\left(X^{\star} X\right)^{-1 / 2}\left(\rho^{2} I-Z^{2}\right)^{-1 / 2}$. Then $\mathcal{F}(X, B)$ is a Hamiltonian matrix such that $\mathcal{F}(X, B) X=B$ and $\|\mathcal{F}(X, B)\|_{2}=\eta_{2}(X, B)$.

In order to construct a real Hamiltonian matrix $\mathcal{H}$ satisfying $\mathcal{H} X=B$ we need the following lemma.

Lemma 3.3. Let $A, B \in \mathbb{C}^{n, p}$. Then $\left[\begin{array}{ll}A & \bar{A}\end{array}\right]\left[\begin{array}{ll}B & \bar{B}\end{array}\right]^{+}$is a real matrix.

Proof. Let $P=\left[\begin{array}{ll}0 & I \\ I & 0\end{array}\right] \in \mathbb{R}^{2 p, 2 p}$. Then $\left[\begin{array}{ll}A & \bar{A}\end{array}\right] P=\left[\begin{array}{ll}\bar{A} & A\end{array}\right]$. Since $P^{-1}=P^{\star}=P$, we have $P\left[\begin{array}{ll}B & \bar{B}\end{array}\right]^{+}=\left(\left[\begin{array}{ll}B & \bar{B}\end{array}\right] P\right)^{+}=\left[\begin{array}{ll}\bar{B} & B\end{array}\right]^{+}$. Hence $\overline{\left[\begin{array}{ll}A & \bar{A}\end{array}\right]\left[\begin{array}{ll}B & \bar{B}\end{array}\right]^{+}}=$ $\overline{\left[\begin{array}{ll}A & \bar{A}\end{array}\right] P^{2}\left[\begin{array}{ll}B & \bar{B}\end{array}\right]^{+}}=\left[\begin{array}{ll}A & \bar{A}\end{array}\right]\left[\begin{array}{ll}B & \bar{B}\end{array}\right]^{+}$.

We then have the following minimal real perturbations.

Corollary 3.4. Let $B \in \mathbb{C}^{2 n, p}, X \in \mathbb{C}^{2 n, p}$, and suppose that $\operatorname{rank}\left[\begin{array}{ll}X & \bar{X}\end{array}\right]=2 p$. Then there exists a real Hamiltonian matrix $\mathcal{H} \in \mathbb{R}^{2 n, 2 n}$ such that $\mathcal{H} X=B$ if and only if $X^{\star} \mathcal{J} B$ is Hermitian and $X^{\star} \mathcal{J} \bar{B}$ is symmetric, i.e., $\left(X^{\star} \mathcal{J} \bar{B}\right)^{\top}=X^{\star} \mathcal{J} \bar{B}$.

If the latter two conditions are satisfied, then with $\mathcal{G}$ as defined in (3.5), the matrix $\mathcal{G}_{\mathbb{R}}:=\mathcal{G}\left(\left[\begin{array}{ll}X & \bar{X}\end{array}\right],\left[\begin{array}{ll}B & \bar{B}\end{array}\right]\right)$ is a real Hamiltonian matrix with $\mathcal{G}_{\mathbb{R}} X=B$. Furthermore, among all real Hamiltonian matrices $\mathcal{H}$ with $\mathcal{H} X=B$ the matrix $\mathcal{G}_{\mathbb{R}}$ has the smallest Frobenius norm.

Proof. If $\mathcal{H}$ is any real matrix with $\mathcal{H} X=B$, then also $\mathcal{H} \bar{X}=\bar{B}$. Hence $\mathcal{H}\left[\begin{array}{ll}X & \bar{X}\end{array}\right]=\left[\begin{array}{ll}B & \bar{B}\end{array}\right]$. By Theorem 3.2 a Hamiltonian matrix $\mathcal{H}$ satisfying this relation exists if and only if $\left[\begin{array}{cc}X & \bar{X}\end{array}\right]^{\star} \mathcal{J}\left[\begin{array}{cc}B & \bar{B}\end{array}\right]=: Z$ is Hermitian. It is easily verified that $Z$ is Hermitian if and only if $X^{\star} \mathcal{J} B$ is Hermitian and $X^{\star} \mathcal{J} \bar{B}$ is symmetric. If these conditions are satisfied, then by Theorem 3.2 the matrix $\mathcal{G}_{\mathbb{R}}$ is Hamiltonian and $\mathcal{G}_{\mathbb{R}}\left[\begin{array}{ll}X & \bar{X}\end{array}\right]=$ $\left[\begin{array}{ll}B & \bar{B}\end{array}\right]$. Moreover, among all Hamiltonian matrices $\mathcal{H}$ with $\mathcal{H}\left[\begin{array}{ll}X & \bar{X}\end{array}\right]=\left[\begin{array}{ll}B & \bar{B}\end{array}\right]$ the matrix $\mathcal{G}_{\mathbb{R}}$ has the smallest Frobenius norm. The realness of $\mathcal{G}_{\mathbb{R}}$ follows from Lemma 3.3 .

In this section we have discussed the structured mapping theorem for Hamiltonian matrices and used it to construct solutions of minimal Frobenius and spectral norms. In the next section we use these results to construct Hamiltonian perturbations that move eigenvalues away from the imaginary axis.

4. Moving eigenvalues by small perturbations. We now discuss in detail how to move an eigenvalue (resp., a group of eigenvalues) of a Hamiltonian matrix by a small Hamiltonian perturbation to a specific location (resp., locations) in the complex plane. We construct Hamiltonian perturbations under the assumption that a $\mathcal{J}$-neutral eigenvector (resp., $\mathcal{J}$-neutral invariant subspace) exists corresponding to the eigenvalue (resp., group of eigenvalues).

THEOREM 4.1. Let $\sigma$ be a set of eigenvalues of a Hamiltonian matrix $\mathcal{H} \in \mathbb{C}^{2 n, 2 n}$, and let $X \in \mathbb{C}^{2 n, d}$ be a full column rank matrix such that $X^{\star} \mathcal{J} X=0$ and $\mathcal{H} X=X R$ for some $R \in \mathbb{C}^{d, d}$ with $\Lambda(R)=\sigma$. Then for any $D \in \mathbb{C}^{d, d}$, the matrix $\mathcal{E}=\mathcal{G}(X, X D)$, where $\mathcal{G}(\cdot, \cdot)$ is defined by (3.5), has the following properties.

(i) The matrix $\mathcal{E}$ is Hamiltonian and satisfies $\mathcal{E}=X D X^{+}+\mathcal{J}\left(X^{+}\right)^{\star} D^{\star} X^{\star} \mathcal{J}$,

Copyright @ $@$ by SIAM. Unauthorized reproduction of this article is prohibited. 
$\mathcal{E} X=X D,\|\mathcal{E}\|_{2}=\left\|X D\left(X^{\star} X\right)^{-1 / 2}\right\|_{2}$, and $\|\mathcal{E}\|_{F}=\sqrt{2}\left\|X D\left(X^{\star} X\right)^{-1 / 2}\right\|_{F}$. Further, we have

$$
(\mathcal{H}+t \mathcal{E}) X=X(R+t D)
$$

for all $t \in \mathbb{R}$, i.e., $\Lambda(R+t D) \subset \Lambda(\mathcal{H}+t \mathcal{E})$ for all $t \in \mathbb{R}$.

(ii) Suppose that $\mathcal{H}$ is real and $\sigma \subset \mathbb{R}$. Then the matrix $X$ can be chosen to be real so that $\mathcal{E}$ is a real Hamiltonian matrix when $D$ is real.

(iii) Suppose that $\mathcal{H}$ is real and $\sigma \not \subset \mathbb{R}$. Set $\bar{\sigma}:=\{\bar{\lambda}: \lambda \in \sigma\}$, and assume that $\sigma \cap$ $\bar{\sigma}=\varnothing$ and $\sigma \cap(-\sigma)=\varnothing$. Then the matrix $\mathcal{K}=\mathcal{G}\left(\left[\begin{array}{ll}X & \bar{X}\end{array}\right],\left[\begin{array}{ll}X D & \overline{X D}\end{array}\right]\right)$ is real Hamiltonian and satisfies $\mathcal{K} X=X D$. Further, for all $t \in \mathbb{R}$ we have

$$
(\mathcal{H}+t \mathcal{K}) X=X(R+t D),
$$

i.e., $\Lambda(R+t D) \subset \Lambda(\mathcal{H}+t \mathcal{K})$ for all $t \in \mathbb{R}$.

Proof. Since $X^{\star} \mathcal{J}(X D)=0$ is Hermitian, by Theorem $3.2, \mathcal{E}$ is a well defined Hamiltonian matrix, $\mathcal{E}=X D X^{+}+\mathcal{J}\left(X^{+}\right)^{\star} D^{\star} X^{\star} \mathcal{J}, \mathcal{E} X=X D,\|\mathcal{E}\|_{2}=\left\|X D\left(X^{\star} X\right)^{-1 / 2}\right\|_{2}$, and $\|\mathcal{E}\|_{F}=\sqrt{2}\left\|X D\left(X^{\star} X\right)^{-1 / 2}\right\|_{F}$. This proves (i).

The assertion in (ii) is obvious. So, suppose that $\mathcal{H}$ is real and $\sigma \not \subset \mathbb{R}$. Then we have $\mathcal{H} X=X R$ and $\mathcal{H} \bar{X}=\bar{X} \bar{R}$ with $\Lambda(R) \cap \Lambda(\bar{R})=\varnothing$. Hence $\operatorname{rank}\left[\begin{array}{ll}X & \bar{X}\end{array}\right]=2 d$. Since $\sigma \cap(-\sigma)=\varnothing$, by Theorem 2.1 the spaces spanned by the columns of $X$ and $\bar{X}$ are $\mathcal{J}$-orthogonal. Thus $X^{\star} \mathcal{J} \bar{X}=0$. As $X^{\star} \mathcal{J} X D=0$ is Hermitian and $X^{\star} \mathcal{J} \overline{X D}=0$ is symmetric, by Corollary 3.4, the matrix $\mathcal{K}$ is real and Hamiltonian with $\mathcal{K} X=X D$. This proves (iii).

Theorem 4.1 shows that an eigenvalue (resp., a group of eigenvalues) of a Hamiltonian matrix $\mathcal{H}$ can be moved by a small Hamiltonian perturbation if the eigenvalue (resp., group of eigenvalues) is associated with a $\mathcal{J}$-neutral eigenvector (resp., $\mathcal{J}$-neutral $\mathcal{H}$-invariant subspace).

Remark 4.2. First, observe that if $\sigma \subset i \mathbb{R}$, then $\bar{\sigma}=-\sigma$ and hence the second assumption in Theorem 4.1(iii) is redundant. Second, if $\lambda \in \mathbb{C} \backslash i \mathbb{R}$ is a nonimaginary eigenvalue of $\mathcal{H}$ and $v$ is an associated eigenvector, then $v$ is $\mathcal{J}$-neutral, that is, $v^{\star} \mathcal{J} v=0$. Thus by Theorem 4.1, a nonimaginary eigenvalue of $\mathcal{H}$ can be moved in any direction in the complex plane by a small Hamiltonian perturbation. More generally, let $\sigma$ be a set of eigenvalues of $\mathcal{H}$ such that $\sigma \subset \mathbb{C}_{-}$(or equivalently $\sigma \subset \mathbb{C}_{+}$). Then by Corollary 2.2, there is a full column rank matrix $X$ such that $X^{\star} \mathcal{J} X=0$ and $\mathcal{H} X=X R$ with $\Lambda(R)=$ $\sigma$ for some matrix $R$. Hence by Theorem 4.1, the group of eigenvalues $\sigma$ can be moved en block by a small Hamiltonian perturbation. Moreover, when $\mathcal{H}$ is real and $\sigma \cap \bar{\sigma}=\varnothing$, then the Hamiltonian perturbation can be chosen to be real.

In view of Remark 4.2 we conclude that a nonimaginary eigenvalue (that is, an eigenvalue with nonzero real part) of a Hamiltonian matrix can be moved in any direction in the complex plane by a small Hamiltonian perturbation. However, this property does not hold in the same generality for purely imaginary eigenvalues. Indeed, suppose that $i \alpha$ is an imaginary eigenvalue of $\mathcal{H}$ and $v$ is an associated eigenvector, i.e., $\mathcal{H} v=i \alpha v$. Then by the Hamiltonian eigenvalue symmetry, $\mathcal{J} v$ is a left eigenvector of $\mathcal{H}$ corresponding to $i \alpha$, i.e., $(\mathcal{J} v)^{\star} \mathcal{H}=i \alpha(\mathcal{J} v)^{\star}$. Thus if $v$ is $\mathcal{J}$-neutral, then $(\mathcal{J} v)^{\star} v=$ $-v^{\star} \mathcal{J} v=0$. Hence it follows that the algebraic multiplicity of $i \alpha$ must be at least 2 . However, the algebraic multiplicity being at least 2 is not enough to remove an imaginary eigenvalue $i \alpha$ from the imaginary axis by a small Hamiltonian perturbation. By "removing" we mean that the perturbed matrix has no imaginary eigenvalue in a vicinity of $i \alpha$. The crux of the matter is that the existence of a $\mathcal{J}$-neutral eigenvector is both a 
necessary and sufficient condition for moving an eigenvalue of a Hamiltonian matrix in any direction in the complex plane by a small Hamiltonian perturbation. More generally, we have the following result.

THeOREM 4.3. Let $\sigma:=\left\{\lambda_{1}, \ldots, \lambda_{d}\right\}$ be a set of distinct eigenvalues in the closed left half-plane of a Hamiltonian matrix $\mathcal{H} \in \mathbb{C}^{2 n, 2 n}$, and let $\mathcal{S}_{\sigma}$ denote the generalized eigenspace $\mathcal{S}_{\sigma}=\bigoplus_{k=1}^{d} \operatorname{ker}\left(\mathcal{H}-\lambda_{k} I\right)^{2 n}$. Let $p$ be any integer with $d \leq p \leq \operatorname{dim} \mathcal{S}_{\sigma}$. Then there exists a Hamiltonian matrix $\mathcal{E}$ such that $\mathcal{H}(t):=\mathcal{H}+t \mathcal{E}$ has a p-dimensional $\mathcal{H}(t)$ invariant subspace $\mathcal{X}(t)$ with $\sigma(t):=\Lambda(\mathcal{H}(t) \mid \mathcal{X}(t)) \subset \mathbb{C}_{-}$for $0<t \leq 1$ and $\sigma(t) \rightarrow \sigma$ as $t \rightarrow 0$ if and only if the subspace $\mathcal{S}_{\sigma}$ contains a $p$-dimensional $\mathcal{J}$-neutral $\mathcal{H}$-invariant subspace $\mathcal{X}$ with $\Lambda(\mathcal{H} \mid \mathcal{X})=\sigma$.

Proof. Suppose that $\mathcal{H} X=X R$ with $\Lambda(R)=\sigma$ and $X^{\star} \mathcal{J} X=0$, where $X \in \mathbb{C}^{2 n, p}$ is a full column rank matrix. Then the desired result follows from Theorem 4.1.

Conversely, suppose that there exists a Hamiltonian matrix $\mathcal{E}$ such that $\mathcal{H}(t):=$ $\mathcal{H}+t \mathcal{E}$ has a $p$-dimensional $\mathcal{H}(t)$-invariant subspace $\mathcal{X}(t)$ with $\sigma(t):=\Lambda(\mathcal{H}(t) \mid \mathcal{X}(t)) \subset$ $\mathbb{C}_{-}$for $0<t \leq 1$ and $\sigma(t) \rightarrow \sigma$ as $t \rightarrow 0$. Let $X(t) \in \mathbb{C}^{2 n, p}$ be a matrix with orthonormal columns such that $\operatorname{span}(X(t))=\mathcal{X}(t)$. Then $\mathcal{H}(t) X(t)=X(t) R(t)$ for some $R(t)$ with $\Lambda(R(t))=\sigma(t)$. By multiplying the former equation from the left with $X(t)^{\star}$, it follows that $R(t)=X(t)^{\star} \mathcal{H}(t) X(t)$. Since for $t>0$, the set $\sigma(t)$ contains no purely imaginary eigenvalue of $\mathcal{H}(t)$, the invariant subspace $\mathcal{X}(t)$ is $\mathcal{J}$-neutral by Corollary 2.2. Thus $X(t)^{\star} \mathcal{J} X(t)=0$ for $t>0$. Since the set of $2 n$-by- $p$ matrices with orthonormal columns is compact, the limit $X=\lim _{k \rightarrow \infty} X\left(t_{k}\right)$ exists for some sequence $\left\{t_{k}\right\}$ with $t_{k} \rightarrow 0$. By continuity, it follows that $X^{\star} \mathcal{J} X=0$ and $\mathcal{H} X=X R$, where $R=\lim _{k \rightarrow \infty} R\left(t_{k}\right)$. Furthermore, $\Lambda(R)=\lim \sigma\left(t_{k}\right)=\sigma$. Hence $\mathcal{X}:=\operatorname{span}(X)$ is a $\mathcal{J}$-neutral $\mathcal{H}$-invariant $p$-dimensional subspace of $\mathcal{S}_{\sigma}$ with $\Lambda(\mathcal{H} \mid \mathcal{X})=\sigma$.

Corollary 4.4. An eigenvalue $\lambda$ of a Hamiltonian matrix $\mathcal{H}$ can be removed from the imaginary axis by an arbitrarily small Hamiltonian perturbation if and only if $\mathcal{H}$ has a $\mathcal{J}$-neutral eigenvector corresponding to $\lambda$.

We mention that an imaginary eigenvalue of a Hamiltonian matrix may or may not have a $\mathcal{J}$-neutral eigenvector associated with it. The case when an imaginary eigenvalue does not have an associated $\mathcal{J}$-neutral eigenvector is addressed in section 5 . In our algorithmic construction we remove one imaginary eigenvalue at a time. Therefore, we first briefly discuss the removal from the imaginary axis of an imaginary eigenvalue by a Hamiltonian perturbation under the assumption that a $\mathcal{J}$-neutral eigenvector exists, and then we discuss how to achieve this property. We have the following result which follows from Theorem 4.1.

THEOREM 4.5. Let $i \alpha$ be an imaginary eigenvalue of a Hamiltonian matrix $\mathcal{H} \in \mathbb{C}^{2 n, 2 n}$. Let $v$ be a normalized and $\mathcal{J}$-neutral eigenvector of $\mathcal{H}$ corresponding to $i \alpha$, i.e., $\|v\|_{2}=1, v^{\star} \mathcal{J} v=0$, and $\mathcal{H} v=i \alpha v$. For any $\mu \in \mathbb{C}$, consider the matrices

$$
\mathcal{E}_{\mu}=\mathcal{G}(v, \mu v) \quad \text { and } \quad \mathcal{K}_{\mu}=\mathcal{G}\left(\left[\begin{array}{ll}
v & \bar{v}
\end{array}\right],\left[\begin{array}{ll}
\mu v & \overline{\mu v}
\end{array}\right]\right),
$$

where $\mathcal{G}(\cdot, \cdot)$ is defined by (3.5). Then $\mathcal{E}_{\mu}$ and $\mathcal{K}_{\mu}$ have the following properties.

(i) The matrix $\mathcal{E}_{\mu}$ is Hamiltonian and satisfies $\mathcal{E}_{\mu}=\mu v v^{\star}+\bar{\mu} \mathcal{J} v v^{\star} \mathcal{J}$, $\left\|\mathcal{E}_{\mu}\right\|_{2}=|\mu|$, and $\left\|\mathcal{E}_{\mu}\right\|_{F}=\sqrt{2}|\mu|$. Furthermore, $\left(\mathcal{H}+t \mathcal{E}_{\mu}\right) v=(i \alpha+t \mu) v$ for all $t \in \mathbb{R}$, i.e., $i \alpha+t \mu \in \Lambda\left(\mathcal{H}+t \mathcal{E}_{\mu}\right)$ for all $t \in \mathbb{R}$.

(ii) If $\mathcal{H}$ is a real matrix and $\alpha=0$, then the vector $v$ can be chosen to be real in which case $\mathcal{E}_{\mu}$ is real for all $\mu \in \mathbb{R}$.

(iii) Suppose that $\mathcal{H}$ is a real matrix and $\alpha \neq 0$. Then $\mathcal{K}_{\mu}$ is a real Hamiltonian matrix satisfying $\left(\mathcal{H}+t \mathcal{K}_{\mu}\right) v=(i \alpha+t \mu) v$ and $\left(\mathcal{H}+t \mathcal{K}_{\mu}\right) \bar{v}=(-i \alpha+t \bar{\mu}) \bar{v}$. Hence $\{i \alpha+t \mu,-i \alpha+t \bar{\mu}\} \subset \Lambda\left(\mathcal{H}+t \mathcal{K}_{\mu}\right)$ for all $t \in \mathbb{R}$.

Copyright () by SIAM. Unauthorized reproduction of this article is prohibited. 
For a purely imaginary eigenvalue with an associated $\mathcal{J}$-neutral eigenvector, the perturbations $\mathcal{E}_{\mu}$ and $\mathcal{K}_{\mu}$ constructed in Theorem 4.5 move the imaginary eigenvalue away from the imaginary axis. Note, however, that these perturbations may also move the other eigenvalues of $\mathcal{H}$ to unspecified positions. For our algorithmic construction, it is desirable to move eigenvalues one-by-one without affecting the other eigenvalues. The following result provides Hamiltonian perturbations which move only the specified eigenvalue and leave the other eigenvalues unchanged.

THEOREM 4.6. Let $i \alpha$ be an imaginary eigenvalue of a Hamiltonian matrix $\mathcal{H} \in \mathbb{C}^{2 n, 2 n}$. Let $v$ be a normalized and $\mathcal{J}$-neutral eigenvector of $\mathcal{H}$ corresponding to $i \alpha$, i.e., $\|v\|_{2}=1, v^{\star} \mathcal{J} v=0$, and $\mathcal{H} v=i \alpha v$. Let $w \in \operatorname{ker}(\mathcal{H}-i \alpha I)^{2 n}$ be such that $w^{\star} \mathcal{J} v=1$. For any $\mu \in \mathbb{C}$, consider the matrices

$$
\hat{\mathcal{E}}_{\mu}=\left(\mu v w^{\star}+\bar{\mu} w v^{\star}\right) \mathcal{J} \quad \text { and } \quad \hat{\mathcal{K}}_{\mu}=\hat{\mathcal{E}}_{\mu}+\overline{\hat{\mathcal{E}}_{\mu}} .
$$

Then $\hat{\mathcal{E}}_{\mu}$ and $\hat{\mathcal{K}}_{\mu}$ have the following properties.

(i) The matrix $\hat{\mathcal{E}}_{\mu}$ is Hamiltonian and $\left(\mathcal{H}+t \hat{\mathcal{E}}_{\mu}\right) v=(i \alpha+t \mu) v$ for all $t \in \mathbb{R}$. Furthermore, $\left(\mathcal{H}+t \hat{\mathcal{E}}_{\mu}\right) x=\mathcal{H} x$ for any $x \in \operatorname{ker}(\mathcal{H}-\lambda I)^{2 n}$ and $\lambda \in$ $\Lambda(\mathcal{H}) \backslash\{i \alpha\}$.

(ii) Suppose that $\mathcal{H}$ is a real matrix and $\alpha=0$. Then the vectors $v$ and $w$ can be chosen to be real in which case $\hat{\mathcal{E}}_{\mu}$ is real for all $\mu \in \mathbb{R}$.

(iii) Suppose that $\mathcal{H}$ is a real matrix and $\alpha \neq 0$. Then the matrix $\hat{\mathcal{K}}_{\mu}$ is a real Hamiltonian matrix satisfying $\left(\mathcal{H}+t \hat{\mathcal{K}}_{\mu}\right) v=(i \alpha+t \mu) v,\left(\mathcal{H}+t \hat{\mathcal{K}}_{\mu}\right) \bar{v}=$ $(-i \alpha+t \bar{\mu}) \bar{v}$, and $\left(\mathcal{H}+t \hat{\mathcal{K}}_{\mu}\right) x=\mathcal{H} x$ for any $x \in \operatorname{ker}(\mathcal{H}-\lambda I)^{2 n}$ and $\lambda \in$ $\Lambda(\mathcal{H}) \backslash\{i \alpha,-i \alpha\}$.

Proof. Since the Hermitian form $(x, y) \mapsto-i x^{\star} \mathcal{J} y$ is nondegenerate on $\operatorname{ker}(\mathcal{H}-i \alpha I)^{2 n}$, there exists $w \in \operatorname{ker}(\mathcal{H}-i \alpha I)^{2 n}$ such that $w^{\star} \mathcal{J} v=1$. Hence $\hat{\mathcal{E}}_{\mu}$ is well defined. Obviously, $\hat{\mathcal{E}}_{\mu} v=\mu v$, whence $\left(\mathcal{H}+t \mathcal{E}_{\mu}\right) v=(i \alpha+t \mu) v$. Since $\operatorname{ker}(\mathcal{H}-i \alpha I)^{2 n}$ is $\mathcal{J}$-orthogonal to the other generalized eigenspaces of $\mathcal{H}$, we have $v^{\star} \mathcal{J} x=w^{\star} \mathcal{J} x=0$ for any $x \in \operatorname{ker}(\mathcal{H}-\lambda I)^{2 n}$ and $\lambda \in \Lambda(\mathcal{H}) \backslash\{i \alpha\}$. Thus $\hat{\mathcal{E}}_{\mu} x=0$. This completes the proof of (i). Assertion (ii) is obvious, and (iii) follows from the identity $\operatorname{ker}(\mathcal{H}+i \alpha I)^{2 n}=$ $\overline{\operatorname{ker}(\mathcal{H}-i \alpha I)^{2 n}}$ and the $\mathcal{J}$-orthogonality of the generalized eigenspaces.

For the construction of Hamiltonian matrices that move eigenvalues off the imaginary axis, we need a $\mathcal{J}$-neutral eigenvector. We now address the issue of existence of $\mathcal{J}$-neutral eigenvectors corresponding to an imaginary eigenvalue of a Hamiltonian matrix. First, we show that a $\mathcal{J}$-neutral eigenvector of $\mathcal{H}$ corresponding to an imaginary eigenvalue exists if the eigenvalue is defective.

Proposition 4.7. Suppose that $v_{1}, v_{2}, \ldots, v_{\ell}, \ell \geq 2$, is a Jordan chain of the Hamiltonian matrix $\mathcal{H}$ associated with an imaginary eigenvalue $i \alpha$, i.e., $\mathcal{H} v_{k}=i \alpha v_{k}+v_{k-1}$ for $k=1, \ldots, \ell$, where $v_{0}:=0$. Then the subspace $\operatorname{span}\left\{v_{1}, \ldots, v_{\lfloor\ell / 2\rfloor}\right\}$ is $\mathcal{J}$-neutral. In particular the eigenvector $v_{1}$ is $\mathcal{J}$-neutral.

Proof. We have $\mathcal{J}(\mathcal{H}-i \alpha I)=-(\mathcal{H}-i \alpha I)^{\star} \mathcal{J}, \quad v_{k}=(\mathcal{H}-i \alpha I)^{\ell-k} v_{\ell} \quad$ for $k=1, \ldots, \ell$, and $(\mathcal{H}-i \alpha I)^{q} v_{\ell}=0$ for $q \geq \ell$. Hence if $k+j \leq \ell$, then $v_{j}^{\star} \mathcal{J} v_{k}=$ $v_{\ell^{\star}}\left((\mathcal{H}-i \alpha I)^{\star}\right)^{\ell-j} \mathcal{J}(\mathcal{H}-i \alpha I)^{\ell-k} v_{\ell}=(-1)^{\ell-j} v_{\ell^{\star}} \mathcal{J}(\mathcal{H}-i \alpha I)^{2 \ell-k-j} v_{\ell}=0$

Proposition 4.7 shows that the first vector in a Jordan chain of length at least 2 is a $\mathcal{J}$-neutral vector, but this may or may not be true for semisimple purely imaginary eigenvalues. To characterize when this is the case, we need the sign characteristic of the purely imaginary eigenvalue, which allows us to classify the purely imaginary eigenvalues into three distinct groups.

Copyright ( by SIAM. Unauthorized reproduction of this article is prohibited. 
DefinITION 4.8. Let ia be a purely imaginary eigenvalue of a nonreal $\mathcal{H} \in \mathbb{C}^{2 n, 2 n}$ or a nonzero purely imaginary eigenvalue of $\mathcal{H} \in \mathbb{R}^{2 n, 2 n}$. Let $X$ be a full column rank matrix such that $\operatorname{span}(X)=\operatorname{ker}\left((\mathcal{H}-i \alpha I)^{2 n}\right)$. Consider the matrix $Z:=-i X^{\star} \mathcal{J} X$. Then i $\alpha$ is said to have positive sign characteristic, negative sign characteristic, or mixed sign characteristic, depending on whether $Z$ is positive definite, negative definite, or indefinite, respectively.

Remark 4.9. Note that the eigenvalue 0 of a real Hamiltonian matrix is excluded in Definition 4.8 because in such a case the definition of sign characteristic does not make sense. Indeed, if 0 is an eigenvalue of a real Hamiltonian matrix and $x$ is an associated eigenvector, then obviously $x^{T} \mathcal{J} x=0$. This shows that there always exists a $\mathcal{J}$-neutral eigenvector of a real Hamiltonian matrix associated with the eigenvalue 0 .

The following result characterizes the existence of a $\mathcal{J}$-neutral eigenvector of a Hamiltonian matrix corresponding to an imaginary eigenvalue; see also [12] in the context of $H$-self-adjoint matrices.

Proposition 4.10. Let i $\alpha$ be a purely imaginary eigenvalue of a nonreal $\mathcal{H} \in \mathbb{C}^{2 n, 2 n}$ or a nonzero purely imaginary eigenvalue of $\mathcal{H} \in \mathbb{R}^{2 n, 2 n}$. Then $\mathcal{H}$ has a $\mathcal{J}$-neutral eigen-

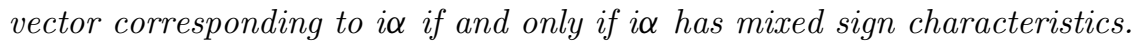

Proof. Recall that the Hermitian form $(x, y) \mapsto-i x^{\star} \mathcal{J} y$ is nondegenerate on $\operatorname{ker}(\mathcal{H}-i \alpha I)^{2 n}$ and hence the matrix $Z=-i X^{\star} \mathcal{J} X$ in Definition 4.8 is nonsingular. Suppose that there exists a $\mathcal{J}$-neutral eigenvector associated with $i \alpha$. Then clearly $Z$ is indefinite. Hence $i \alpha$ has mixed sign characteristics.

Conversely, suppose that $i \alpha$ has mixed sign characteristics; i.e., $Z$ is indefinite. By Proposition 4.7, a $\mathcal{J}$-neutral eigenvector exists if the eigenvalue $i \alpha$ is defective. So, suppose that $i \alpha$ is semisimple. Since $Z$ is indefinite, there exist eigenvectors $v_{0}$ and $v_{1}$ such that $-i v_{0}^{\star} \mathcal{J} v_{0}>0$ and $-i v_{1}^{\star} \mathcal{J} v_{1}<0$. Hence by continuity there exists an eigenvector $v$ of the form $v=\cos (t) v_{0}+\sin (t) v_{1}$, for some $t \in \mathbb{R}$, such that $v^{\star} \mathcal{J} v=0$.

Note that if a purely imaginary eigenvalue of a nonreal Hamiltonian matrix or a nonzero purely imaginary eigenvalue of a real Hamiltonian matrix is simple, then it has either positive or negative sign characteristic. Hence if $i \alpha$ has mixed sign characteristics, then $i \alpha$ is necessarily multiple. Note, further, that if $i \alpha$ is defective, then by Proposition 4.7, $i \alpha$ has mixed sign characteristics. However, when $i \alpha$ is a nondefective multiple eigenvalue, it may or may not have mixed sign characteristics; see [28, Example 6].

Remark 4.11. We have shown that only eigenvalues of mixed sign characteristics can be removed from the imaginary axis by an arbitrarily small Hamiltonian perturbation. A related result is well-known for symplectic perturbations of eigenvalues of symplectic matrices on the unit circle; see [39, p. 196].

5. Minimal Hamiltonian perturbations. In this section we investigate how to move purely imaginary eigenvalues which are neither defective nor have mixed sign characteristics off the imaginary axis by suitable Hamiltonian perturbations. We begin with the problem of moving an eigenvalue of a Hamiltonian matrix to a specified point in the complex plane by a minimal Hamiltonian perturbation. This will play an important role in moving eigenvalues to specific points outside a strip $S_{\tau}$ as required in Problem B.

By the previous discussion, in order to move a purely imaginary eigenvalue having either positive or negative sign characteristic from the imaginary axis by a Hamiltonian perturbation, we first need to coalesce it with another purely imaginary eigenvalue of opposite sign characteristic.

Thus in this case we split the construction of perturbations that move the eigenvalues off the imaginary axis into two steps. First, we construct a minimal Hamiltonian

Copyright ( by SIAM. Unauthorized reproduction of this article is prohibited. 
perturbation that coalesces two eigenvalues having negative and positive sign characteristics into an imaginary eigenvalue having mixed sign characteristics. This moves the eigenvalues on the boundary of the set required in Problem A. Second, we move the resulting imaginary eigenvalue with mixed sign characteristics off the imaginary axis by a small Hamiltonian perturbation as required in Problem B.

Since we have already addressed the second stage of the problem in the previous section, we now address the first step of the construction.

For this purpose, we make use of both the backward error for the Hamiltonian eigenvalue problem and Hamiltonian pseudospectra. These quantities are introduced and discussed in the following subsections. In the third subsection we then determine perturbations of minimum norm which remove a pair of eigenvalues from the imaginary axis.

5.1. Backward errors. We begin with the construction of backward errors for eigenvalues of a Hamiltonian matrix. The Hamiltonian backward error associated with a complex number $\lambda \in \mathbb{C}$ is defined by

$$
\eta^{\mathrm{Ham}}(\lambda, \mathcal{H}):=\inf \left\{\|\mathcal{E}\|: \mathcal{E} \in \mathbb{F}^{2 n, 2 n} \text { Hamiltonian, } \lambda \in \Lambda(\mathcal{H}+\mathcal{E})\right\}
$$

Note that in general $\eta(\lambda, \mathcal{H})$ will be different for $\mathbb{F}=\mathbb{C}$ and for $\mathbb{F}=\mathbb{R}$. We use the notation $\eta_{F}^{\mathrm{Ham}}(\lambda, \mathcal{H})$ and $\eta_{2}^{\mathrm{Ham}}(\lambda, \mathcal{H})$ when the norm in (5.1) is the Frobenius norm and the spectral norm, respectively.

Theorem 5.1. Let $\mathcal{H} \in \mathbb{C}^{2 n, 2 n}$ be a Hamiltonian matrix, and let $\lambda \in \mathbb{C}$ be such that $\operatorname{Re} \lambda \neq 0$. Then we have

$$
\begin{aligned}
& \eta_{F}^{\mathrm{Ham}}(\lambda, \mathcal{H})=\min _{\|x\|_{2}=1}\left\{\sqrt{2\|(\mathcal{H}-\lambda I) x\|_{2}^{2}-\left|x^{\star} \mathcal{J H} x\right|^{2}} \quad: x \in \mathbb{C}^{2 n}, x^{\star} \mathcal{J} x=0\right\}, \\
& \eta_{2}^{\mathrm{Ham}}(\lambda, \mathcal{H})=\min _{\|x\|_{2}=1}\left\{\|(\mathcal{H}-\lambda I) x\|_{2}: x \in \mathbb{C}^{2 n}, x^{\star} \mathcal{J} x=0\right\} .
\end{aligned}
$$

In particular, we have $\eta_{2}^{\mathrm{Ham}}(\lambda, \mathcal{H}) \leq \eta_{F}^{\mathrm{Ham}}(\lambda, \mathcal{H}) \leq \sqrt{2} \eta_{2}^{\mathrm{Ham}}(\lambda, \mathcal{H})$.

Suppose that the minima in (5.2) and (5.3) are attained for $u \in \mathbb{C}^{2 n}$ and $v \in \mathbb{C}^{2 n}$, respectively. Let $\mathcal{E}:=\mathcal{G}(u,(\lambda I-\mathcal{H}) u)$ and $\mathcal{K}:=\mathcal{F}(v,(\lambda I-\mathcal{H}) v)$, where $\mathcal{G}$ and $\mathcal{F}$ are as in Theorem 3.2. Then

$$
\begin{aligned}
& \|\mathcal{E}\|_{F}=\eta_{F}^{\mathrm{Ham}}(\lambda, \mathcal{H}) \quad \text { and } \quad(\mathcal{H}+\mathcal{E}) u=\lambda u, \\
& \|\mathcal{K}\|_{2}=\eta_{2}^{\operatorname{Ham}}(\lambda, \mathcal{H}) \quad \text { and } \quad(\mathcal{H}+\mathcal{K}) v=\lambda v .
\end{aligned}
$$

Proof. Let $x \in \mathbb{C}^{n}$ be nonzero. Then by Theorem 3.2 there exists a Hamiltonian matrix $\mathcal{E} \in \mathbb{C}^{2 n, 2 n}$ such that $(\mathcal{H}+\mathcal{E}) x=\lambda x$ if and only if $x^{\star} \mathcal{J} x=0$. Indeed, setting $r=\lambda x-\mathcal{H} x$, it follows that $x^{\star} \mathcal{J} r$ is real if and only if $x^{\star} \mathcal{J} x=0$. So, suppose that $x^{\star} \mathcal{J} x=0$ and w.l.o.g. that $x^{\star} x=1$. Then by Theorem $3.2, \mathcal{E}:=\mathcal{G}(x, r)$ is the unique Hamiltonian matrix such that $(\mathcal{H}+\mathcal{E}) x=\lambda x$ and $\mathcal{E}$ has minimal Frobenius norm given by

$$
\|\mathcal{E}\|_{F}=\sqrt{2\|(\mathcal{H}-\lambda I) x\|_{2}^{2}-\left|x^{\star} \mathcal{J}(\mathcal{H}-\lambda I) x\right|^{2}} .
$$

Similarly, by Theorem $3.2, \mathcal{K}:=\mathcal{F}(x, r)$ is a Hamiltonian matrix such that $(\mathcal{H}+\mathcal{K}) x=\lambda x$ and $\mathcal{K}$ has minimal spectral norm given by

Copyright () by SIAM. Unauthorized reproduction of this article is prohibited. 


$$
\|\mathcal{K}\|_{2}=\|(\mathcal{H}-\lambda I) x\|_{2}
$$

The claim follows by taking the minimum over all $x \in \mathbb{C}^{2 n}$ such that $x^{\star} \mathcal{J} x=0$.

Note that it is a nontrivial task to determine the minimal values $\eta_{2}^{\text {Ham }}(\lambda, \mathcal{H})$ and $\eta_{F}^{\mathrm{Ham}}(\lambda, \mathcal{H})$ when $\lambda \in \mathbb{C}$ and $\operatorname{Re} \lambda \neq 0$. In contrast, it is relatively simple to determine these minimal values for purely imaginary values $\lambda=i \omega$ with $\omega \in \mathbb{R}$. The construction in Proposition 5.3 below is based on the following observation.

Observation 5.2. Let $\mathcal{H} \in \mathbb{C}^{2 n, 2 n}$ be Hamiltonian, and let $\lambda_{1}, \ldots, \lambda_{2 n} \in \mathbb{R}$ denote the eigenvalues of the Hermitian matrix $\mathcal{J H}$. Let $v_{1}, \ldots, v_{2 n} \in \mathbb{C}^{2 n}$ be an orthonormal basis of eigenvectors of $\mathcal{J H}$ such that $\mathcal{J H} v_{k}=\lambda_{k} v_{k}$. Then $\left|\lambda_{1}\right|, \ldots,\left|\lambda_{2 n}\right|$ are the singular values of $\mathcal{H}$, and the vectors $v_{k}$ are the associated right singular vectors. The associated left singular vectors are $u_{k}=-\operatorname{sign}\left(\lambda_{k}\right) \mathcal{J} v_{k}$. Indeed, the matrices $V=\left[v_{1}, \ldots, v_{2 n}\right]$, $U=\left[u_{1}, \ldots, u_{2 n}\right]$ are unitary, and from $\mathcal{J H} V=V \operatorname{diag}\left(\lambda_{1}, \ldots, \lambda_{2 n}\right)$ it follows that $\mathcal{H}=U \operatorname{diag}\left(\left|\lambda_{1}\right|, \ldots,\left|\lambda_{2 n}\right|\right) V^{\star}$.

In the following we denote the smallest singular value of a matrix $M$ by $\sigma_{\min }(M)$.

Proposition 5.3. Let $\mathcal{H} \in \mathbb{F}^{2 n, 2 n}$ be Hamiltonian and $\omega \in \mathbb{R}$. Let $v$ be a normalized eigenvector of the Hermitian matrix $\mathcal{J}(\mathcal{H}-i \omega I)$ corresponding to an eigenvalue $\lambda \in \mathbb{R}$. Then $|\lambda|$ is a singular value of the Hamiltonian matrix $\mathcal{H}-i \omega I$, and $v$ is an associated right singular vector.

Further, the matrices

$$
\begin{aligned}
\mathcal{E} & =\lambda \mathcal{J} v v^{\star}, \\
\mathcal{K} & =\lambda \mathcal{J}[v \bar{v}][v \bar{v}]^{+}
\end{aligned}
$$

are Hamiltonian, $\mathcal{K}$ is real, and we have $(\mathcal{H}+\mathcal{E}) v=(\mathcal{H}+\mathcal{K}) v=i \omega v$. Furthermore, $\|\mathcal{E}\|_{F}=\|\mathcal{E}\|_{2}=\|\mathcal{K}\|_{2}=|\lambda|$ and $\|\mathcal{K}\|_{F}=|\lambda| \sqrt{\operatorname{rank}\left[\begin{array}{ll}v & \bar{v}\end{array}\right]}$.

Moreover, suppose that $\lambda$ is an eigenvalue of $\mathcal{J}(\mathcal{H}-i \omega I)$ of smallest absolute value and let $\sigma_{\min }(\mathcal{H}-i \omega I)$ be the smallest singular value of $\mathcal{H}-i \omega I$. Then $|\lambda|=$ $\sigma_{\min }(\mathcal{H}-i \omega I)$, and we have

$$
\begin{aligned}
& \eta_{F}^{\mathrm{Ham}}(i \omega, \mathcal{H})=\eta_{2}^{\mathrm{Ham}}(i \omega, \mathcal{H})=|\lambda|=\|\mathcal{E}\|_{2} \quad \text { when } \quad \mathbb{F}=\mathbb{C}, \\
& \eta_{F}^{\mathrm{Ham}}(i \omega, \mathcal{H}) \leq\|\mathcal{K}\|_{F} \leq \sqrt{2} \eta_{2}^{\text {Ham }}(i \omega, \mathcal{H})=\sqrt{2}|\lambda|=\sqrt{2}\|\mathcal{K}\|_{2} \quad \text { when } \quad \mathbb{F}=\mathbb{R} .
\end{aligned}
$$

Proof. The first assertion follows by applying Observation 5.2 to the Hamiltonian matrix $\mathcal{H}-i \omega I$. By construction, $\mathcal{H}$ and $\mathcal{K}$ are Hamiltonian and $(\mathcal{H}+\mathcal{E}) v=$ $(\mathcal{H}+\mathcal{K}) v=i \omega v$. Note that by Lemma $3.3, \mathcal{K}$ is real. Obviously, we have $\|\mathcal{E}\|_{F}=\|\mathcal{E}\|_{2}=$ $\|\mathcal{K}\|_{2}=|\lambda|$ and $\|\mathcal{K}\|_{F}=|\lambda| \sqrt{\operatorname{rank}\left[\begin{array}{ll}v & \bar{v}\end{array}\right]}$.

If $\lambda$ has the smallest absolute value, then $\sigma_{\min }(\mathcal{H}-i \omega I)=|\lambda|$ by Observation 5.2. Since $\frac{1}{\sqrt{2}}\|\mathcal{K}\|_{F} \leq\|\mathcal{K}\|_{2}=\|\mathcal{E}\|_{F}=\|\mathcal{E}\|_{2}=\sigma_{\text {min }}(\mathcal{H}-i \omega I)$ and $\eta_{F}^{\text {Ham }}(i \omega, \mathcal{H}) \geq \eta_{2}^{\text {Ham }}(i \omega, \mathcal{H}) \geq$ $\sigma_{\text {min }}(\mathcal{H}-i \omega I)$, the desired result follows.

Proposition 5.3 in particular states that a Hamiltonian perturbation of $\mathcal{H}$ of smallest norm that moves an eigenvalue to the point $i \omega$ can be constructed from an eigenpair $(v, \lambda)$ of $\mathcal{J}(\mathcal{H}-i \omega I)$, where $\lambda$ has the smallest absolute value. Our next result shows that the eigenpair $(v, \lambda)$ can be chosen as a piecewise analytic (but not necessarily continuous) function of $\omega$.

Proposition 5.4. Let $\mathcal{H} \in \mathbb{C}^{2 n, 2 n}$ be Hamiltonian, and let $F(\omega)=\mathcal{J}(\mathcal{H}-i \omega I)$ and $f(\omega)=\sigma_{\min }(\mathcal{H}-i \omega I)$ for $\omega \in \mathbb{R}$. There exist a finite number $\ell$ of real values $\gamma_{1}<\gamma_{2}<$ $\cdots<\gamma_{\ell}$ and functions $\lambda_{\min }: \mathbb{R} \rightarrow \mathbb{R}, v: \mathbb{R} \rightarrow \mathbb{C}^{2 n}$ which are analytic on $\mathbb{R} \backslash\left\{\gamma_{1}, \ldots, \gamma_{\ell}\right\}$

Copyright ( by SIAM. Unauthorized reproduction of this article is prohibited. 
and have the following properties.

(a) $F(\omega) v(\omega)=\lambda_{\min }(\omega) v(\omega),\left|\lambda_{\min }(\omega)\right|=\min \{|\lambda|: \lambda \in \Lambda(F(\omega))\}$, and, moreover, $\|v(\omega)\|_{2}=1$ for all $\omega \in \mathbb{R}$.

(b) For each $k \in\{0,1, \ldots, \ell\}$ either $\lambda_{\min }(\omega)=f(\omega)$ for all $\omega \in\left(\gamma_{k}, \gamma_{k+1}\right)$ or $\lambda_{\min }(\omega)=-f(\omega)$ for all $\omega \in\left(\gamma_{k}, \gamma_{k+1}\right)$, where we set $\gamma_{0}=-\infty$ and $\gamma_{\ell+1}=\infty$.

(c) The vector $v(\omega)$ is a right singular vector of the matrix $\mathcal{H}-i \omega I$ associated with the smallest singular value.

(d) The derivative of $\lambda_{\min }(\cdot)$ at $\omega \in \mathbb{R} \backslash\left\{\gamma_{1}, \ldots, \gamma_{\ell}\right\}$ satisfies

$$
\lambda_{\min }^{\prime}(\omega)=-i v(\omega)^{\star} \mathcal{J} v(\omega) .
$$

(e) At each of the (exceptional) points $\gamma_{k}$ the left and the right limits of $\lambda_{\min }(\cdot)$ and $v(\cdot)$ exist. Suppose that $\lambda_{\min }(\cdot)$ is continuous at $\gamma_{k}$. Then the left and the right side derivative of $\lambda_{\min }(\cdot)$ at $\gamma_{k}$ both exist and satisfy

$$
\lim _{\omega \rightarrow \gamma_{k} \pm} \frac{\lambda_{\min }(\omega)-\lambda_{\min }\left(\gamma_{k}\right)}{\omega-\gamma_{k}}=\lim _{\omega \rightarrow \gamma_{k} \pm} \lambda_{\min }^{\prime}(\omega)
$$

Proof. Note that $F(\omega)=\mathcal{J}(\mathcal{H}-i \omega I), \omega \in \mathbb{R}$, is a Hermitian matrix. By [33, pp. 29-33] there exist analytic functions $\omega \mapsto v_{1}(\omega), \ldots, v_{2 n}(\omega) \in \mathbb{C}^{2 n}$ and $\omega \mapsto \lambda_{1}(\omega), \ldots, \lambda_{2 n}(\omega) \in \mathbb{R}$ such that for each $\omega$ the vectors $v_{j}(\omega)$ form an orthonormal basis of $\mathbb{C}^{2 n}$ and $F(\omega) v_{j}(\omega)=\lambda_{j}(\omega) v_{j}(\omega)$. The derivative of $\lambda_{j}$ at $\omega$ satisfies

$$
\begin{aligned}
\lambda_{j}^{\prime}(\omega) & =\frac{d}{d \omega}\left(v_{j}(\omega)^{\star} F(\omega) v_{j}(\omega)\right) \\
& =v_{j}(\omega)^{\star} F^{\prime}(\omega) v_{j}(\omega)+v_{j}^{\prime}(\omega)^{\star} F(\omega) v_{j}(\omega)+v_{j}(\omega)^{\star} F(\omega) v_{j}^{\prime}(\omega) \\
& =-i v_{j}(\omega)^{\star} \mathcal{J} v_{j}(\omega)+\lambda_{j}(\omega) \underbrace{\left(v_{j}^{\prime}(\omega)^{\star} v_{j}(\omega)+v_{j}(\omega)^{\star} v_{j}^{\prime}(\omega)\right)}_{=\frac{d}{d \omega}\left\|v_{j}(\omega)\right\|^{2}=0} \\
& =-i v_{j}(\omega)^{\star} \mathcal{J} v_{j}(\omega) .
\end{aligned}
$$

For each pair of indices $j, k$ the analytic functions $\lambda_{j}(\cdot), \lambda_{k}(\cdot)$ either are identical or meet in a discrete set $P_{j, k} \subset \mathbb{R}$. Analogously, the functions $-\lambda_{j}(\cdot), \lambda_{k}(\cdot)$ either are identical or meet in a discrete set $Q_{j, k} \subset \mathbb{R}$. Since the union of the graphs of the functions $\pm \lambda_{j}(\cdot)$ equals the algebraic curve $\left\{(\omega, \lambda) \in \mathbb{R}^{2} \mid \operatorname{det}((F(\omega)-\lambda I)(F(\omega)+\lambda I))=0\right\}$, both of the sets $P_{j, k}$ and $Q_{j, k}$ are finite [3]. Let $\left\{\gamma_{1}, \ldots, \gamma_{r}\right\}, \gamma_{k}<\gamma_{k+1}$, denote the union of the sets $P_{j k}$ and the sets $Q_{j k}$. By the third claim of Proposition 5.3, we have that $f(\omega)=$ $\min _{j=1, \ldots, 2 n}\left|\lambda_{j}(\omega)\right|$. It follows that to each interval $\mathcal{I}_{k}=\left(\gamma_{k}, \gamma_{k+1}\right)$ there exists an index $j$ such that either $\lambda_{j}(\omega)=f(\omega)$ for all $\omega \in \mathcal{I}_{k}$ or $\lambda_{j}(\omega)=-f(\omega)$ for all $\omega \in \mathcal{I}_{k}$. Define $\lambda_{\min }(\omega):=\lambda_{j}(\omega), v(\omega):=v_{j}(\omega)$ for $\omega \in \mathcal{I}_{k}$ and $\lambda_{\min }\left(\gamma_{k}\right):=\lambda_{j}\left(\gamma_{k}\right), v\left(\gamma_{k}\right):=v_{j}\left(\gamma_{k}\right)$. Then the functions $\lambda_{\min }(\cdot)$ and $v(\cdot)$ have the required properties.

Example 5.5. The left diagram of Figure 1 shows the eigenvalue curves $\omega \mapsto \lambda_{j}(\omega)$ of the Hermitian matrix function $\omega \mapsto \mathcal{J}\left(\mathcal{H}_{1}-i \omega I\right)$ for $\omega \in[-16,16]$ and $\mathcal{H}_{1}:=\left[\begin{array}{cc}0 & G_{1} \\ H_{1} & 0\end{array}\right]$, where

Copyright ( by SIAM. Unauthorized reproduction of this article is prohibited. 

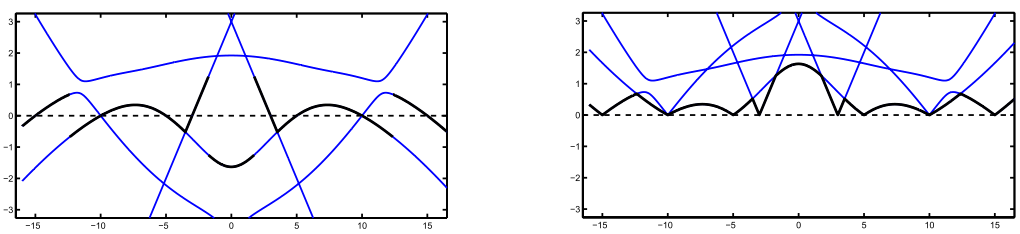

FIG. 5.1. Eigenvalue and singular value curves for Example 5.5.

$$
G_{1}:=\left[\begin{array}{ccccc}
7 & -4 & 2 & -11 & 0 \\
-4 & -37 & 31 & -8 & 0 \\
2 & 31 & -28 & 4 & 0 \\
-11 & -8 & 4 & 28 & 0 \\
0 & 0 & 0 & 0 & -3
\end{array}\right], \quad H_{1}:=\left[\begin{array}{ccccc}
11 & 16 & 16 & 5 & 0 \\
16 & 21 & 30 & 8 & 0 \\
16 & 30 & 48 & 8 & 0 \\
5 & 8 & 8 & -1 & 0 \\
0 & 0 & 0 & 0 & 3
\end{array}\right] .
$$

The spectrum of $\mathcal{H}_{1}$ is $\Lambda\left(\mathcal{H}_{1}\right)=\{ \pm 3 i, \pm 5 i \pm 10 i, \pm 15 i\}$, and the eigenvalues $\pm 10 i$ have multiplicity 2 , while the other eigenvalues are simple. At the real parts of the eigenvalues of $\mathcal{H}_{1}$ the eigenvalue curves $\lambda_{j}(\cdot)$ cross the real axis. Observe that, according to (5.6), the sign characteristics of the eigenvalues of $\mathcal{H}_{1}$ can be read off from the slopes of the curves $\lambda_{j}(\cdot)$. The $\lambda_{j}$-curves crossing the real axis at $-15,-3$, and 5 have positive slope; i.e., the eigenvalues $-15 i,-3 i$, and $5 i$ have positive sign characteristic. The $\lambda_{j}(\cdot)$-curves crossing the real axis at $-5,3$, and 15 have negative slope; i.e., the eigenvalues $-5 i, 3 i$, and $15 i$ have negative sign characteristic. At the points \pm 10 there are two $\lambda_{j}$-curves crossing the real axis with positive and negative slopes. Thus the eigenvalues \pm 10 both have mixed sign characteristic. The graph of the function $\omega \mapsto \lambda_{\min }(\omega)=\lambda_{\min }\left(J\left(\mathcal{H}_{1}-i \omega I\right)\right)$ from Proposition 5.4 is depicted by thick curves. Note that this function is piecewise analytic but discontinuous. The right diagram of Figure 1 shows the singular value curves of the pencil $\omega \mapsto \mathcal{H}_{1}-i \omega I$. The graph of the continuous function $\omega \mapsto \sigma_{\min }\left(\mathcal{H}_{1}-i \omega I\right)$ is depicted as a thick curve. Note that $\sigma_{\min }\left(\mathcal{H}_{1}-i \omega I\right)=\left|\lambda_{\min }(\omega)\right|$.

The following proposition characterizes the existence of $\mathcal{J}$-neutral eigenvectors in terms of the local extrema of the eigenvalue curves.

Proposition 5.6. Suppose the function $\lambda_{\min }: \mathbb{R} \rightarrow \mathbb{R}$ of Proposition 5.4 is continuous at $\omega_{0} \in \mathbb{R}$ and attains a local extremum at $\omega_{0}$. Then there exists a $\mathcal{J}$-neutral normalized eigenvector $v_{0}$ of the Hermitian matrix $\mathcal{J}\left(\mathcal{H}-i \omega_{0} I\right)$ corresponding to the eigenvalue $\lambda_{\min }\left(\omega_{0}\right)$.

Proof. If $\omega_{0} \in \mathbb{R} \backslash\left\{\gamma_{1}, \ldots, \gamma_{r}\right\}$, then the derivative of $\lambda_{\min }(\cdot)$ at $\omega_{0}$ satisfies $0=$ $\lambda_{\text {min }}^{\prime}\left(\omega_{0}\right)=-i v\left(\omega_{0}\right)^{\star} \mathcal{J} v\left(\omega_{0}\right)$. Hence $v_{0}:=v\left(\omega_{0}\right)$ is $\mathcal{J}$-neutral if $\lambda_{\min }$ attains a local extremum at $\omega_{0}$. Now suppose that $\omega_{0} \in\left\{\gamma_{1}, \ldots, \gamma_{r}\right\}$. Assume w.l.o.g. that $\omega_{0}$ is a local maximum. Then the left sided derivative of $\lambda_{\min }(\cdot)$ at $\omega_{0}$ is nonnegative, and the right sided derivative is nonpositive. Hence it follows from claim (e) of Proposition 5.4 that

$$
\begin{aligned}
& 0 \leq \lim _{\omega \rightarrow \omega_{0}-} \lambda_{\text {min }}^{\prime}(\omega)=\lim _{\omega \rightarrow \omega_{0}-}\left(-i v(\omega)^{\star} \mathcal{J} v(\omega)\right)=-i v_{-}^{\star} \mathcal{J} v_{-}, \\
& 0 \geq \lim _{\omega \rightarrow \omega_{0}+} \lambda^{\prime}(\omega)=\lim _{\omega \rightarrow \omega_{0}-}\left(-i v(\omega)^{\star} \mathcal{J} v(\omega)\right)=-i v_{+}^{\star} \mathcal{J} v_{+},
\end{aligned}
$$

where $v_{ \pm}=\lim _{\omega \rightarrow \omega_{0} \pm} v(\omega)$. Suppose that $v_{+}$and $v_{-}$are linearly dependent. Then $-i v_{-}^{\star} \mathcal{J} v_{-}=-i v_{+}^{\star} \mathcal{J} v_{+}=0$; i.e., $v_{0}:=v_{+}$has the required properties. If $v_{+}$and $v_{-}$ are linearly independent, then let $u_{t}=t v_{+}+(1-t) v_{-}$. In this case for all $t \in \mathbb{R}, u_{t} \neq$ 0 and $\mathcal{J}\left(\mathcal{H}-i \omega_{0} I\right) u_{t}=\lambda_{\text {min }}\left(\omega_{0}\right) u_{t}$. Furthermore, $-i u_{0}^{\star} \mathcal{J} u_{0} \leq 0$ and $-i u_{1}^{\star} \mathcal{J} u_{1} \geq 0$. By continuity there exists $t_{0} \in[0,1]$ such that $-i u_{t_{0}}^{\star} \mathcal{J} u_{t_{0}}=0$, and hence $v_{0}:=u_{t_{0}} /\left\|u_{t_{0}}\right\|$ has the required properties.

Copyright ( by SIAM. Unauthorized reproduction of this article is prohibited. 
5.2. Pseudospectra. Let $A \in \mathbb{C}^{n, n}$, and let $\epsilon \geq 0$. Then the $\epsilon$-pseudospectrum of $A$ is defined as

$$
\Lambda_{\epsilon}(A, \mathbb{F})=\bigcup_{\|E\|_{2} \leq \epsilon}\left\{\Lambda(A+E): E \in \mathbb{F}^{n, n}\right\}
$$

It is well-known [38] that in the complex case when $\mathbb{F}=\mathbb{C}$, we have

$$
\Lambda_{\epsilon}(A, \mathbb{C})=\left\{z \in \mathbb{C}: \sigma_{\min }(A-z I) \leq \epsilon\right\}
$$

where, as noted above, $\sigma_{\min }(\cdot)$ denotes the minimum singular value. Since we are interested in structured perturbations, we also consider the Hamiltonian $\epsilon$-pseudospectrum defined by

$$
\Lambda_{\epsilon}^{\operatorname{Ham}}(\mathcal{H}, \mathbb{F})=\bigcup_{\|\mathcal{E}\|_{2} \leq \epsilon}\left\{\Lambda(\mathcal{H}+\mathcal{E}): \mathcal{E} \in \mathbb{F}^{2 n, 2 n} \text { and }(\mathcal{J E})^{\star}=\mathcal{J E}\right\}
$$

It is obvious that

$$
\Lambda_{\epsilon}^{\operatorname{Ham}}(\mathcal{H}, \mathbb{C})=\left\{z \in \mathbb{C}: \eta_{2}^{\operatorname{Ham}}(z, \mathcal{H}) \leq \epsilon\right\},
$$

where $\eta_{2}^{\operatorname{Ham}}(z, \mathcal{H})$ is the Hamiltonian backward error as defined in (5.1). Note that the pseudospectra so defined will in general be different for $\mathbb{F}=\mathbb{C}$ and for $\mathbb{F}=\mathbb{R}$; however, for purely imaginary eigenvalues, the following result is an immediate consequence of Proposition 5.3.

Corollary 5.7. Let $\mathcal{H} \in \mathbb{C}^{2 n, 2 n}$ be Hamiltonian. Consider the pseudospectra $\Lambda_{\epsilon}(\mathcal{H} ; \mathbb{F})$ and $\Lambda_{\epsilon}^{\mathrm{Ham}}(\mathcal{H} ; \mathbb{F})$. Then

$$
\begin{aligned}
\Lambda_{\epsilon}^{\mathrm{Ham}}(\mathcal{H} ; \mathbb{C}) \cap i \mathbb{R} & =\Lambda_{\epsilon}^{\mathrm{Ham}}(\mathcal{H} ; \mathbb{R}) \cap i \mathbb{R}=\Lambda_{\epsilon}(\mathcal{H} ; \mathbb{C}) \cap i \mathbb{R}=\Lambda_{\epsilon}(\mathcal{H} ; \mathbb{R}) \cap i \mathbb{R} \\
& =\left\{i \omega: \omega \in \mathbb{R}, \sigma_{\min }(\mathcal{H}-i \omega I) \leq \epsilon\right\} \\
& =\left\{i \omega: \omega \in \mathbb{R},\left|\lambda_{\min }(\mathcal{J}(\mathcal{H}-i \omega I))\right| \leq \epsilon\right\},
\end{aligned}
$$

where $\lambda_{\min }(\cdot)$ denotes the eigenvalue function from Proposition 5.4 .

In Definition 4.8 we have associated sign characteristics to the purely imaginary eigenvalues of a Hamiltonian matrix. We now associate sign characteristics to the connected components of a Hamiltonian pseudospectrum.

Definition 5.8. Let $\mathcal{H} \in \mathbb{F}^{2 n, 2 n}$. A connected component $\mathcal{C}_{\epsilon}(\mathcal{H})$ of $\Lambda_{\epsilon}^{\operatorname{Ham}}(\mathcal{H}, \mathbb{F})$ is said to have positive (resp., negative) sign characteristic if for all Hamiltonian perturbations $\mathcal{E}$ with $\|\mathcal{E}\|_{2} \leq \epsilon$ each eigenvalue of $\mathcal{H}+\mathcal{E}$ that is contained in $\mathcal{C}_{\epsilon}(\mathcal{H})$ has positive (resp., negative) sign characteristic.

Observe that if a component $\mathcal{C}_{\epsilon}(\mathcal{H})$ of $\Lambda_{\epsilon}^{\mathrm{Ham}}(\mathcal{H}, \mathbb{F})$ has positive (resp., negative) sign characteristic, then $\mathcal{C}_{\epsilon}(\mathcal{H}) \subset i \mathbb{R}$ and all eigenvalues of $\mathcal{H}$ that are contained in $\mathcal{C}_{\epsilon}(\mathcal{H})$ have positive (resp., negative) sign characteristic. We now show that the sign characteristic of $\mathcal{C}_{\epsilon}(\mathcal{H})$ is completely determined by the sign characteristic of the eigenvalues of $\mathcal{H}$ that are contained in $\mathcal{C}_{\epsilon}(\mathcal{H})$.

Theorem 5.9. Let $\mathcal{H} \in \mathbb{F}^{2 n, 2 n}$, and let $\mathcal{C}_{\epsilon}(\mathcal{H})$ be a connected component of $\Lambda_{\epsilon}^{\mathrm{Ham}}(\mathcal{H}, \mathbb{F})$. For a Hamiltonian matrix $\mathcal{E} \in \mathbb{F}^{2 n, 2 n}$ with $\|\mathcal{E}\|_{2} \leq \epsilon$, let $X_{\mathcal{E}}$ be a full column rank matrix whose columns form a basis of the direct sum of the generalized eigenspaces $\operatorname{ker}(\mathcal{H}+\mathcal{E}-\lambda I)^{2 n}, \lambda \in \mathcal{C}_{\epsilon}(\mathcal{H}) \cap \Lambda(\mathcal{H}+\mathcal{E})$. Set $Z_{\mathcal{E}}:=-i X_{\mathcal{E}}^{\star} \mathcal{J} X_{\mathcal{E}}$. Then the following conditions are equivalent.

Copyright ( by SIAM. Unauthorized reproduction of this article is prohibited. 
(a) The component $\mathcal{C}_{\epsilon}(\mathcal{H})$ has positive (resp., negative) sign characteristic.

(b) All eigenvalues of $\mathcal{H}$ that are contained in $\mathcal{C}_{\epsilon}(\mathcal{H})$ have positive (resp., negative) sign characteristic.

(c) The matrix $Z_{0}$ associated with $\mathcal{E}=0$ is positive (resp., negative) definite.

(d) The matrix $Z_{\mathcal{E}}$ is positive (resp., negative) definite for all Hamiltonian matrix $\mathcal{E}$ with $\|\mathcal{E}\|_{2} \leq \epsilon$.

Proof. Now w.l.o.g. suppose that $\mathcal{C}_{\epsilon}(\mathcal{H})$ has positive sign characteristic. Then obviously all eigenvalues of $\mathcal{H}$ that are contained in $\mathcal{C}_{\epsilon}(\mathcal{H})$ have positive sign characteristic. This proves $(\mathrm{a}) \Rightarrow(\mathrm{b})$.

Next, suppose that $\Lambda(\mathcal{H}) \cap \mathcal{C}_{\epsilon}(\mathcal{H})$ contains $p$ distinct eigenvalues $i \alpha_{1}, \ldots, i \alpha_{p}$ each of which has positive sign characteristic. Let $X_{k}$ be a full column rank matrix whose columns form a basis of $\operatorname{ker}\left(\mathcal{H}-i \alpha_{k}\right)^{2 n}$ for $k=1, \ldots, p$. Then the columns of $\mathcal{J} X_{k}$ form a basis of the left generalized eigenspace of $\mathcal{H}$ corresponding to the eigenvalue $i \alpha_{k}$. Hence $X_{k}^{\star} \mathcal{J} X_{l}=-\left(\mathcal{J} X_{k}\right)^{\star} X_{l}=0$ for $l \neq k$. Since $i \alpha_{k}$ has positive sign characteristic, the matrix $-i X_{k}^{\star} \mathcal{J} X_{k}$ is positive definite for $k=1, \ldots, p$. Now considering $X:=\left[X_{1}, \ldots, X_{p}\right]$ it follows that $-i X^{\star} \mathcal{J} X=\operatorname{diag}\left(-i X_{1}^{\star} \mathcal{J} X_{1}, \ldots,-i X_{p}^{\star} \mathcal{J} X_{p}\right)$ is positive definite. Since $X_{0}=X M$ for some nonsingular matrix $M$, it follows that $Z_{0}$ is congruent to $-i X^{\star} \mathcal{J} X$. Hence $Z_{0}$ is positive definite. This proves $(\mathrm{b}) \Rightarrow(\mathrm{c})$.

Now suppose that $Z_{0}$ is positive definite. Since $\mathcal{C}_{\epsilon}(\mathcal{H})$ is a closed and connected component of $\Lambda_{\epsilon}^{\operatorname{Ham}}(\mathcal{H}, \mathbb{F})$, there is a simple closed rectifiable curve $\Gamma$ such that $\Gamma \cap \Lambda_{\epsilon}^{\operatorname{Ham}}(\mathcal{H}, \mathbb{F})=\varnothing$ and such that the component $\mathcal{C}_{\epsilon}(\mathcal{H})$ lies inside the curve $\Gamma$. Let $\mathcal{E}$ be a Hamiltonian matrix with $\|\mathcal{E}\|_{2} \leq \epsilon$. Consider the matrix $\mathcal{H}(t):=\mathcal{H}+t \mathcal{E}$ for $t \in \mathbb{C}$. Then by [20, Chapters II.3-II.4, pp. 66-68] there exists a matrix $X_{\mathcal{E}}(t)$ such that $X_{\mathcal{E}}(t)$ is analytic in $D_{\Gamma}:=\left\{t \in \mathbb{C}:|t|\|\mathcal{E}\|_{2}<\min _{z \in \Gamma} \sigma_{\min }(\mathcal{H}-z I)\right\}$. Further, for each $t \in$ $D_{\Gamma}$ the matrix $X_{\mathcal{E}}(t)$ has full column rank and the columns form a basis of the direct sum of the generalized eigenspaces $\operatorname{ker}(\mathcal{H}(t)-\lambda I)^{2 n}, \lambda \in \Lambda(\mathcal{H}(t)) \cap \mathcal{C}_{\epsilon}(\mathcal{H})=: \sigma_{\mathcal{E}}(t)$. Since $\|\mathcal{E}\|_{2} \leq \epsilon$ and $\min _{z \in \Gamma} \sigma_{\min }(\mathcal{H}-z I)>\epsilon$, it follows that $[0,1] \subset D_{\Gamma}$. Hence the matrix $X_{\mathcal{E}}(t)$ is smooth on $[0,1]$. Set $Z_{\mathcal{E}}(t):=-i X_{\mathcal{E}}(t)^{\star} \mathcal{J} X_{\mathcal{E}}(t)$ for $t \in[0,1]$. Then $Z_{\mathcal{E}}(t)$ is continuous and, by Corollary $2.2, Z_{\mathcal{E}}(t)$ is nonsingular for $t \in[0,1]$. Indeed, since $\sigma_{\mathcal{E}}(t)$ is symmetric with respect to the imaginary axis, the columns of $X_{\mathcal{E}}(t)$ form a basis of the direct sum of the $\mathcal{J}$-nondegenerate and pairwise $\mathcal{J}$-orthogonal subspaces $\operatorname{ker}(\mathcal{H}(t)-$ $i \alpha I)^{2 n}, \quad i \alpha \in \sigma_{\mathcal{E}}(t)$, and $\operatorname{ker}(\mathcal{H}(t)-\lambda I)^{2 n} \bigoplus \operatorname{ker}(\mathcal{H}(t)+\bar{\lambda} I)^{2 n}, \quad \lambda \in \sigma_{\mathcal{E}}(t) \backslash i \mathbb{R} ; \quad$ see Corollary 2.2. It follows that $\operatorname{span}\left(X_{\mathcal{E}}(t)\right)$ is $\mathcal{J}$-nondegenerate. Thus $Z_{\mathcal{E}}(t)$ is nonsingular for all $t \in[0,1]$. Since $Z_{\mathcal{E}}(0)$ is positive definite and $Z_{\mathcal{E}}(t)$ is nonsingular for all $t$ in the connected set $[0,1]$, it follows that $Z_{\mathcal{E}}(t)$ is positive definite for all $t \in[0,1]$. This shows that $Z_{\mathcal{E}}$ is positive definite. Since $\mathcal{E}$ is arbitrary, we conclude that the assertion in (d) holds. This proves $(\mathrm{c}) \Rightarrow(\mathrm{d})$.

Finally, suppose that the assertion in (d) holds. Then obviously for all Hamiltonian matrices $\mathcal{E}$ with $\|\mathcal{E}\|_{2} \leq \epsilon$, the eigenvalues in $\Lambda(\mathcal{H}+\mathcal{E}) \cap \mathcal{C}_{\epsilon}(\mathcal{H})$ are purely imaginary and have positive sign characteristic. In other words, $\mathcal{C}_{\epsilon}(\mathcal{H})$ has positive sign characteristic. This completes the proof.

The following result is an immediate consequence of the proof of Theorem 5.9.

Corollary 5.10. Let $\mathcal{H} \in \mathbb{F}^{2 n, 2 n}$, and let $\mathcal{C}_{\epsilon}(\mathcal{H})$ be a connected component of $\Lambda_{\epsilon}^{\mathrm{Ham}}(\mathcal{H}, \mathbb{F})$. For a Hamiltonian matrix $\mathcal{E} \in \mathbb{F}^{2 n, 2 n}$ with $\|\mathcal{E}\|_{2} \leq \epsilon$, let $X_{\mathcal{E}}$ be a full column rank matrix whose columns form a basis of the direct sum of the generalized eigenspaces $\operatorname{ker}(\mathcal{H}+\mathcal{E}-\lambda I)^{2 n}, \lambda \in \mathcal{C}_{\epsilon}(\mathcal{H}) \cap \Lambda(\mathcal{H}+\mathcal{E})$. Set $Z_{\mathcal{E}}:=-i X_{\mathcal{E}}^{\star} \mathcal{J} X_{\mathcal{E}}$. Then the following assertions hold.

(i) The rank of $X_{\mathcal{E}}$ is constant for all Hamiltonian matrices $\mathcal{E}$ with $\|\mathcal{E}\|_{2} \leq \epsilon$.

(ii) If $\mathcal{C}_{\epsilon}(\mathcal{H}) \cap i \mathbb{R}=\varnothing$, then $Z_{\mathcal{E}}=0$ for all Hamiltonian matrices $\mathcal{E}$ with $\|\mathcal{E}\|_{2} \leq \epsilon$.

Copyright ( $)$ by SIAM. Unauthorized reproduction of this article is prohibited. 
(iii) If $\mathcal{C}_{\epsilon}(\mathcal{H}) \cap i \mathbb{R} \neq \varnothing$, then $\mathcal{C}_{\epsilon}(\mathcal{H})=-\overline{\mathcal{C}_{\epsilon}(\mathcal{H})}$ and $Z_{\mathcal{E}}$ is nonsingular for all Hamiltonian matrices $\mathcal{E}$ with $\|\mathcal{E}\|_{2} \leq \epsilon$. Furthermore, the matrix $\mathcal{Z}_{\mathcal{E}}$ has the same inertia for all such $\mathcal{E}$.

(iv) If $Z_{\mathcal{E}}$ is positive (resp., negative) definite for some Hamiltonian matrix $\mathcal{E}$ with $\|\mathcal{E}\|_{2} \leq \epsilon$, then $\mathcal{C}_{\epsilon}(\mathcal{H}) \subseteq i \mathbb{R}$ and $\mathcal{C}_{\epsilon}(\mathcal{H})$ has positive (resp., negative) sign characteristic.

The results in Theorem 5.9 and Corollary 5.10 provide important insight into the evolution of purely imaginary eigenvalues of a Hamiltonian matrix subject to Hamiltonian perturbations. With a desire to further understand this evolution, we now analyze the coalescence of pseudospectral components.

5.3. Coalescence of pseudospectral components. Consider the Hamiltonian pseudospectrum $\Lambda_{\epsilon}^{\mathrm{Ham}}(\mathcal{H}, \mathbb{F})$ of a Hamiltonian matrix $\mathcal{H} \in \mathbb{F}^{2 n, 2 n}$. Then obviously the set valued map $\epsilon \mapsto \Lambda_{\epsilon}^{\operatorname{Ham}}(\mathcal{H}, \mathbb{F})$ is monotonically increasing; i.e., if $\epsilon<\delta$, then $\Lambda_{\epsilon}^{\mathrm{Ham}}(\mathcal{H}, \mathbb{F}) \subset \Lambda_{\delta}^{\operatorname{Ham}}(\mathcal{H}, \mathbb{F})$. Furthermore, for $\epsilon>0$, the pseudospectrum $\Lambda_{\epsilon}^{\mathrm{Ham}}(\mathcal{H}, \mathbb{F})$ consists of at most $2 n$ connected components, and each component contains at least one eigenvalue of $\mathcal{H}$. Thus when $\epsilon$ is sufficiently small, then each component of $\Lambda_{\epsilon}^{\mathrm{Ham}}(\mathcal{H}, \mathbb{F})$ contains exactly one eigenvalue of $\mathcal{H}$ and as $\epsilon$ increases, these components expand in size and at some stage coalesce with each other. So, let $i \alpha$ be a purely imaginary eigenvalue of $\mathcal{H}$, and let $\mathcal{C}_{\epsilon}(\mathcal{H}, i \alpha)$ denote the connected component of $\Lambda_{\epsilon}^{\mathrm{Ham}}(\mathcal{H}, \mathbb{F})$ which contains $i \alpha$. Then for a sufficiently small $\epsilon, \mathcal{C}_{\epsilon}(\mathcal{H}, i \alpha) \cap \Lambda(\mathcal{H})=\{i \alpha\}$. Thus if $i \alpha$ has either positive or negative sign characteristic, then by Theorem 5.9 we have $\mathcal{C}_{\epsilon}(\mathcal{H}, i \alpha) \subset i \mathbb{R}$. This means that the eigenvalue $i \alpha$ cannot be removed from the imaginary axis by a Hamiltonian perturbation $\mathcal{E}$ of $\mathcal{H}$ such that $\|\mathcal{E}\|_{2} \leq \epsilon$.

Next, let $i \beta$ be another purely imaginary eigenvalue of $\mathcal{H}$ with $\alpha<\beta$, and suppose that $\mathcal{C}_{\epsilon}(\mathcal{H}, i \beta)$ is a component of $\Lambda_{\epsilon}^{\mathrm{Ham}}(\mathcal{H}, \mathbb{F})$ containing $i \beta$ such that $\mathcal{C}_{\epsilon}(\mathcal{H}, i \beta) \cap$ $\Lambda(\mathcal{H})=\{i \beta\}$. Suppose further that $i \beta$ has either positive or negative sign characteristic so that by Theorem 5.9 we have $\mathcal{C}_{\epsilon}(\mathcal{H}, i \beta) \subset i \mathbb{R}$. Assume that $\mathcal{H}$ does not have an eigenvalue $i \gamma$ with $\gamma \in(\alpha, \beta)$ and that the component $\mathcal{C}_{\epsilon}(\mathcal{H}, i \alpha)$ coalesces with the component $\mathcal{C}_{\epsilon}(\mathcal{H}, i \beta)$ at $i \omega_{0}$ as $\epsilon$ tends to $\epsilon_{0}$, i.e., $\mathcal{C}_{\epsilon}(\mathcal{H}, i \alpha) \cap \mathcal{C}_{\epsilon}(\mathcal{H}, i \beta)=\varnothing$ for $\epsilon<\epsilon_{0}$ and $\mathcal{C}_{\epsilon_{0}}(\mathcal{H}, i \alpha) \cap \mathcal{C}_{\epsilon_{0}}(\mathcal{H}, i \beta)=\left\{i \omega_{0}\right\}$. We now investigate the geometry of the connected component $\mathcal{C}_{\epsilon_{0}+\delta}(\mathcal{H}, i \alpha)=\mathcal{C}_{\epsilon_{0}+\delta}(\mathcal{H}, i \beta)$ of $\Lambda_{\epsilon_{0}+\delta}^{\mathrm{Ham}}(\mathcal{H}, \mathbb{F})$ in a neighborhood of $i \omega_{0}$ for a small $\delta>0$. In particular, we show that when $i \alpha$ and $i \beta$ have opposite sign characteristics, then the pseudospectrum $\Lambda_{\epsilon_{0}+\delta}^{\mathrm{Ham}}(\mathcal{H}, \mathbb{F})$ contains a disk centered at $i \omega_{0}$. Furthermore, in this case we show that there exists a Hamiltonian matrix $\mathcal{E}$ with $\|\mathcal{E}\|_{2}=\epsilon_{0}$ such that when $\mathcal{H}$ is perturbed to $\mathcal{H}+\mathcal{E}$, then the eigenvalues $i \alpha$ and $i \beta$ coalesce at $i \omega_{0}$ to form an eigenvalue of $\mathcal{H}+\mathcal{E}$ of mixed sign characteristics. This multiple eigenvalue can then be removed from the imaginary axis by an arbitrarily small Hamiltonian perturbation of $\mathcal{H}+\mathcal{E}$.

We say that two purely imaginary eigenvalues $i \alpha$ and $i \beta$ of $\mathcal{H}$ are adjacent if $\mathcal{H}$ does not have an eigenvalue $i \gamma$ with $\min \{\alpha, \beta\}<\gamma<\max \{\alpha, \beta\}$.

THEOREM 5.11. Let $i \alpha$ and $i \beta$ be adjacent imaginary eigenvalues of a Hamiltonian matrix $\mathcal{H} \in \mathbb{F}^{2 n, 2 n}$ with $\alpha<\beta$. Let $f(\omega):=\sigma_{\min }(\mathcal{H}-i \omega I)$ for $\omega \in \mathbb{R}$, and let $\omega_{0} \in(\alpha, \beta)$ be such that $f\left(\omega_{0}\right)=\max \{f(\omega): \omega \in[\alpha, \beta]\}$. Set $\epsilon_{0}:=f\left(\omega_{0}\right)$. Suppose that the following conditions are satisfied.

(i) For $\epsilon<\epsilon_{0}$ the connected components $\mathcal{C}_{\epsilon}(\mathcal{H}, i \alpha)$ and $\mathcal{C}_{\epsilon}(\mathcal{H}, i \beta)$ of $\Lambda_{\epsilon}^{\mathrm{Ham}}(\mathcal{H}, \mathbb{F})$ containing the eigenvalues $i \alpha$ and $i \beta$, respectively, have either positive or negative sign characteristic.

(ii) If $\omega \in[\alpha, \beta]$, then $i \omega \in \mathcal{C}_{f(\omega)}(\mathcal{H}, i \alpha) \cup \mathcal{C}_{f(\omega)}(\mathcal{H}, i \beta)$. Then the following assertions hold. 
(a) The function $f$ is strictly increasing in $\left[\alpha, \omega_{0}\right]$ and strictly decreasing in $\left[\omega_{0}, \beta\right]$. For $\epsilon<\epsilon_{0}$, we have $i \omega_{0} \notin \Lambda_{\epsilon}^{\mathrm{Ham}}(\mathcal{H}, \mathbb{F}), \mathcal{C}_{\epsilon}(\mathcal{H}, i \alpha) \cap \mathcal{C}_{\epsilon}(\mathcal{H}, i \beta)=\varnothing$, and $i \omega_{0} \in$ $\mathcal{C}_{\epsilon_{0}}(\mathcal{H}, i \alpha)=\mathcal{C}_{\epsilon_{0}}(\mathcal{H}, i \beta)=\mathcal{C}_{\epsilon_{0}}(\mathcal{H}, i \alpha) \cup \mathcal{C}_{\epsilon_{0}}(\mathcal{H}, i \beta)$.

(b) Let $\lambda_{\min }(\cdot)$ be the function given in Proposition 5.4. If i $\alpha$ has positive sign characteristic and $i \beta$ has negative sign characteristic, then $\lambda_{\min }(\omega)=f(\omega)$ for all $\omega \in[\alpha, \beta]$. On the other hand, if $i \alpha$ has negative sign characteristic and $i \beta$ has positive sign characteristic, then $\lambda_{\min }(\omega)=-f(\omega)$ for all $\omega \in[\alpha, \beta]$. In both cases there exists a $\mathcal{J}$-neutral normalized eigenvector $v_{0}$ of $\mathcal{J}\left(\mathcal{H}-i \omega_{0} I\right)$ corresponding to the eigenvalue $\lambda_{\min }\left(\omega_{0}\right)$.

(c) Suppose that the eigenvalues $i \alpha$ and $i \beta$ have opposite sign characteristic. Then for any $\delta>0$ we have $\left\{z \in \mathbb{C}:\left|z-i \omega_{0}\right| \leq \delta\right\} \subset \Lambda_{\epsilon_{0}+\delta}^{\text {Ham }}(\mathcal{H}, \mathbb{F})$ when $\mathbb{F}=\mathbb{C}$. When $\mathbb{F}=\mathbb{R}$ and $\omega_{0} \neq 0$, then for any $\delta>0$ there exists an $\eta>0$ such that $\left\{z \in \mathbb{C}:\left|z-i \omega_{0}\right| \leq \eta\right\} \subset \Lambda_{\epsilon_{0}+\delta}^{\mathrm{Ham}}(\mathcal{H}, \mathbb{F})$. Further, for any $\delta>0$ the interval $[-\delta, \delta]$ is contained in $\Lambda_{\epsilon_{0}+\delta}^{\mathrm{Ham}}(\mathcal{H}, \mathbb{F})$ when $\mathbb{F}=\mathbb{R}$ and $\omega_{0}=0$.

(d) Suppose that the eigenvalues $i \alpha$ and $i \beta$ have the same sign characteristic. Then for $\epsilon \geq \epsilon_{0}, \mathcal{C}_{\epsilon}(\mathcal{H}, i \alpha)$ is a connected component of $\Lambda_{\epsilon}^{\mathrm{Ham}}(\mathcal{H}, \mathbb{F})$ containing the eigenvalues $i \alpha$ and $i \beta$. If $\mathcal{C}_{\epsilon_{0}}(\mathcal{H}, i \alpha)$ contains no other eigenvalues of $\mathcal{H}$ except $i \alpha$ and $i \beta$, then $\mathcal{C}_{\epsilon_{0}}(\mathcal{H}, i \alpha) \subset i \mathbb{R}$ and has the same sign characteristic as that of $i \alpha$. Moreover, in such a case, there is a $\delta_{0}>0$ such that $\mathcal{C}_{\epsilon_{0}+\delta}(\mathcal{H}, i \alpha) \subset i \mathbb{R}$ for all $0 \leq \delta<\delta_{0}$.

Proof. (a) Observe that if $\epsilon<\epsilon_{0}=f\left(\omega_{0}\right)$, then $i \omega_{0} \notin \Lambda_{\epsilon}^{\operatorname{Ham}}(\mathcal{H}, \mathbb{F})$, and hence by assumption (i) and Corollary 5.10 we have that $\mathcal{C}_{\epsilon}(\mathcal{H}, i \alpha) \cap \mathcal{C}_{\epsilon}(\mathcal{H}, i \beta)=\varnothing$ and that $\mathcal{C}_{\epsilon}(\mathcal{H}, i \alpha) \subset i \mathbb{R}$ and $\mathcal{C}_{\epsilon}(\mathcal{H}, i \beta) \subset i \mathbb{R}$. By assumption (ii) it follows that $\mathcal{C}_{\epsilon_{0}}(\mathcal{H}, i \alpha) \cup$ $\mathcal{C}_{\epsilon_{0}}(\mathcal{H}, i \beta)$ is a connected component of $\Lambda_{\epsilon_{0}}^{\mathrm{Ham}}(\mathcal{H}, \mathbb{F})$ and hence $i \omega_{0} \in \mathcal{C}_{\epsilon_{0}}(\mathcal{H}, i \alpha)=$ $\mathcal{C}_{\epsilon_{0}}(\mathcal{H}, i \beta)$.

First, we show that $f$ is strictly increasing in $\left[\alpha, \omega_{0}\right]$. Let $\gamma_{1}, \gamma_{2} \in\left[\alpha, \omega_{0}\right]$ be such that $\gamma_{1}<\gamma_{2}$. Then by assumption (ii), we have $i \gamma_{2} \in \mathcal{C}_{f\left(\gamma_{2}\right)}(\mathcal{H}, i \alpha) \cup \mathcal{C}_{f\left(\gamma_{2}\right)}(\mathcal{H}, i \beta)$. Now, suppose that $f\left(\gamma_{2}\right)<\epsilon_{0}=f\left(\omega_{0}\right)$. Then, as we have just seen, $\mathcal{C}_{f\left(\gamma_{2}\right)}(\mathcal{H}, i \alpha) \cap$ $\mathcal{C}_{f\left(\gamma_{2}\right)}(\mathcal{H}, i \beta)=\varnothing$, and hence $i \gamma_{2} \in \mathcal{C}_{f\left(\gamma_{2}\right)}(\mathcal{H}, i \alpha) \subset i \mathbb{R}$. Let $\mathcal{E} \in \mathbb{F}^{2 n, 2 n}$ be a Hamiltonian matrix such that $\|\mathcal{E}\|_{2}=f\left(\gamma_{2}\right)$ and $i \gamma_{2} \in \Lambda(\mathcal{H}+\mathcal{E})$. Setting $\mathcal{H}(t):=\mathcal{H}+t \mathcal{E}$, it follows that $\Lambda(\mathcal{H}(t)) \subset \Lambda_{f\left(\gamma_{2}\right)}^{\mathrm{Ham}}(\mathcal{H}, \mathbb{F})$ for $t \in[0,1]$. Since $i \alpha \in \Lambda(\mathcal{H}(0))$ and $i \gamma_{2} \in \Lambda(\mathcal{H}(1))$, by the continuity of eigenvalues it follows that $\Lambda(\mathcal{H}(t)) \cap \mathcal{C}_{f\left(\gamma_{2}\right)}(\mathcal{H}, i \alpha) \neq \varnothing$ for $t \in[0,1]$ and that $i \gamma_{1} \in \Lambda\left(\mathcal{H}\left(t_{0}\right)\right)$ for some $t_{0} \in(0,1)$. Hence $f\left(\gamma_{1}\right) \leq\left\|t_{0} \mathcal{E}\right\|_{2}<\|\mathcal{E}\|_{2}=f\left(\gamma_{2}\right)$.

Next, suppose that $f\left(\gamma_{2}\right)=\epsilon_{0}=f\left(\omega_{0}\right)$. If $\gamma_{2}=\omega_{0}$, then there is nothing to prove. So, suppose that $\gamma_{2}<\omega_{0}$. Then there exists $\gamma_{3} \in\left(\gamma_{2}, \omega_{0}\right)$ such that $f\left(\gamma_{3}\right)<f\left(\omega_{0}\right)=\epsilon_{0}$. Since $\gamma_{2}, \gamma_{3} \in\left[\alpha, \omega_{0}\right]$ with $\gamma_{2}<\gamma_{3}$ and $f\left(\gamma_{3}\right)<\epsilon_{0}$, as we have just proved above, we have that $\epsilon_{0}=f\left(\gamma_{2}\right)<f\left(\gamma_{3}\right)$, which is a contradiction. Hence we conclude that $f$ is strictly increasing in $\left[\alpha, \omega_{0}\right]$. By similar arguments, it follows that $f$ is strictly decreasing in $\left[\omega_{0}, \beta\right]$. This concludes the proof of (a).

(b) Note that $f(\alpha)=f(\beta)=0$ and that for any $\omega \in[\alpha, \beta] \backslash\left\{\omega_{0}\right\}$ the connected components $\mathcal{C}_{f(\omega)}(\mathcal{H}, i \alpha)$ and $\mathcal{C}_{f(\omega)}(\mathcal{H}, i \beta)$ are disjoint, and

$$
\begin{aligned}
& i[\alpha, \omega] \subseteq \mathcal{C}_{f(\omega)}(\mathcal{H}, i \alpha) \quad \text { if } \omega \in\left[\alpha, \omega_{0}\right), \\
& i[\omega, \beta] \subseteq \mathcal{C}_{f(\omega)}(\mathcal{H}, i \beta) \quad \text { if } \omega \in\left(\omega_{0}, \beta\right] .
\end{aligned}
$$

Now consider the functions $\lambda_{\min }(\cdot)$ and $v(\cdot)$ given in Proposition 5.4. There exist finitely many numbers $-\infty=\gamma_{0}<\gamma_{1}<\cdots<\gamma_{r}<\gamma_{r+1}=\infty$ and signs $s_{k} \in\{-1,1\}$ such that $\lambda_{\min }(\cdot)$ is analytic on $\left(\gamma_{k}, \gamma_{k+1}\right)$ and $f(\omega)=s_{k} \lambda_{\min }(\omega)$ for $\omega \in\left(\gamma_{k}, \gamma_{k+1}\right)$. Then $\mathcal{E}(\omega)=\lambda_{\min }(\omega) \mathcal{J} v(\omega) v(\omega)^{\star}$ is Hamiltonian, $\|\mathcal{E}(\omega)\|_{2}=f(\omega)$, and $(\mathcal{H}+\mathcal{E}(\omega)) v(\omega)=$ 
$i \omega v(\omega)$. Let $\omega \in\left(\alpha, \omega_{0}\right)$. Then by (5.7) the eigenvalue $i \omega$ of $\mathcal{H}+\mathcal{E}(\omega)$ lies in the connected component $\mathcal{C}_{f(\omega)}(\mathcal{H}, i \alpha)$ which has the same sign characteristic as that of $i \alpha$.

Suppose that $i \alpha$ has positive sign characteristic. Then $\mathcal{C}_{f(\omega)}(\mathcal{H}, i \alpha)$ has positive sign characteristic. Thus $i \omega$ has positive sign characteristic and therefore $-i v(\omega)^{\star} \mathcal{J} v(\omega)>$ 0 . Analogously we have $-i v(\omega)^{\star} \mathcal{J} v(\omega)<0$ for all $\omega \in\left(\omega_{0}, \beta\right]$. Now, for $\omega \in$ $[\alpha, \beta] \backslash\left\{\gamma_{1}, \ldots, \gamma_{r}\right\}$, we have

$$
-s_{k} i v^{\star}(\omega) \mathcal{J} v(\omega)=s_{k} \lambda_{\min }^{\prime}(\omega)=f^{\prime}(\omega) \begin{cases}\geq 0 & \text { if } \omega \in\left[\alpha, \omega_{0}\right] \cap\left(\gamma_{k}, \gamma_{k+1}\right), \\ \leq 0 & \text { if } \omega \in\left[\omega_{0}, \beta\right] \cap\left(\gamma_{k}, \gamma_{k+1}\right) .\end{cases}
$$

The latter inequalities are consequences of (a). It follows that $s_{k}=1$ and hence $f(\omega)=$ $\lambda_{\min }(\omega)$ for all $\omega \in[\alpha, \beta]$. Our derivation of the latter identity was based on the assumption that $i \alpha$ has positive sign characteristic and $i \beta$ has negative sign characteristic. In the opposite case an analogous argument leads to the conclusion that $f(\omega)=-\lambda_{\min }(\omega)$ for all $\omega \in[\alpha, \beta]$. Since $f$ is a continuous function, it now follows from Proposition 5.6 that there exists a $\mathcal{J}$-neutral unit vector $v_{0}$ such that $\mathcal{J}\left(\mathcal{H}-i \omega_{0} I\right) v_{0}=\lambda_{\min }\left(\omega_{0}\right) v_{0}$. This concludes the proof of (b).

(c) Let $\mu \in \mathbb{C}$ and consider $\mathcal{E}:=\lambda_{\min }\left(\omega_{0}\right) \mathcal{J} v_{0} v_{0}^{\star}+\mathcal{G}\left(v_{0}, \mu v_{0}\right)$ when $\mathbb{F}=\mathbb{C}$, where $\mathcal{G}(\cdot, \cdot)$ is defined as in Theorem 3.2. Then $\mathcal{E}$ is Hamiltonian, $(\mathcal{H}+\mathcal{E}) v_{0}=$ $\left(i \omega_{0}+\mu\right) v_{0}$, and $\left\|\mathcal{G}\left(v_{0}, \mu v_{0}\right)\right\|_{2}=|\mu|$. Hence the desired result follows when $\mathbb{F}=\mathbb{C}$.

Note that $v_{0}$ is real when $\mathbb{F}=\mathbb{R}$ and $\omega_{0}=0$. Hence $\mathcal{E}$ is real and Hamiltonian for $\mu \in \mathbb{R}$. Consequently, we have $[-\delta, \delta] \subset \Lambda_{\epsilon_{0}+\delta}^{\mathrm{Ham}}(\mathcal{H}, \mathbb{F})$ when $\mathbb{F}=\mathbb{R}$ and $\omega_{0}=0$.

Now, suppose that $\mathbb{F}=\mathbb{R}$ and $\omega_{0} \neq 0$. Let $\mu \in \mathbb{C}$. Then it is easily seen that $\operatorname{rank}\left[\begin{array}{cc}v_{0} & \bar{v}_{0}\end{array}\right]=2$ and $\left[v_{0} \bar{v}_{0}\right]^{\star} \mathcal{J}\left[\begin{array}{ll}\mu v_{0} & \bar{\mu} \bar{v}_{0}\end{array}\right]=0$. Consider

$$
\mathcal{K}:=\lambda_{\min }\left(\omega_{0}\right) \mathcal{J}\left[\begin{array}{ll}
v_{0} & \bar{v}_{0}
\end{array}\right]\left[\begin{array}{ll}
v_{0} & \bar{v}_{0}
\end{array}\right]^{+}+\mathcal{G}\left(\left[\begin{array}{ll}
v_{0} & \bar{v}_{0}
\end{array}\right],\left[\begin{array}{ll}
\mu v_{0} & \bar{\mu} \bar{v}_{0}
\end{array}\right]\right),
$$

where $\mathcal{G}\left(\left[\begin{array}{ll}v_{0} & \bar{v}_{0}\end{array}\right],\left[\begin{array}{ll}\mu v_{0} & \bar{\mu} \bar{v}_{0}\end{array}\right]\right)=\left[\begin{array}{lll}\mu v_{0} & \bar{\mu} \bar{v}_{0}\end{array}\right]\left[v_{0} \bar{v}_{0}\right]^{+}+\mathcal{J}\left[\begin{array}{ll}\mu v_{0} & \bar{\mu} \bar{v}_{0}\end{array}\right]\left[\begin{array}{ll}v_{0} & \bar{v}_{0}\end{array}\right]^{+} \mathcal{J}$ is defined as in Theorem 3.2. Then $\mathcal{K}$ is real and Hamiltonian, $(\mathcal{H}+\mathcal{K}) v_{0}=\left(i \omega_{0}+\mu\right) v_{0}$, and $\left\|\mathcal{G}\left(\left[\begin{array}{ll}v_{0} & \bar{v}_{0}\end{array}\right],\left[\begin{array}{ll}\mu v_{0} & \bar{\mu} \bar{v}_{0}\end{array}\right]\right)\right\|_{2} \leq 2|\mu|\left\|\left[\begin{array}{ll}v_{0} & \bar{v}_{0}\end{array}\right]\right\|_{2}\left\|\left[v_{0} \bar{v}_{0}\right]^{+}\right\|_{2}$ Hence for $\delta>0$, setting $\eta:=$ $\delta /\left(2\left\|\left[\begin{array}{ll}v_{0} & \bar{v}_{0}\end{array}\right]\right\|_{2}\left\|\left[v_{0} \bar{v}_{0}\right]^{+}\right\|_{2}\right)$, it follows that the disk $\left\{i \omega_{0}+\mu: \mu \in \mathbb{C}:|\mu| \leq \eta\right\}$ is contained in $\Lambda_{\epsilon_{0}+\delta}^{\mathrm{Ham}}(\mathcal{H}, \mathbb{R})$. This proves (c).

(d) Finally, w.l.o.g. suppose that both the eigenvalues $i \alpha$ and $i \beta$ have positive sign characteristic. Then both components $\mathcal{C}_{\epsilon}(\mathcal{H}, i \alpha)$ and $\mathcal{C}_{\epsilon}(\mathcal{H}, \beta)$ have positive sign characteristic for all $\epsilon<\epsilon_{0}$. Hence $\mathcal{C}_{\epsilon}(\mathcal{H}, i \alpha) \cup \mathcal{C}_{\epsilon}(\mathcal{H}, i \beta) \subset i \mathbb{R}$ for all $\epsilon<\epsilon_{0}$. Recall that $\mathcal{C}_{\epsilon_{0}}(\mathcal{H}, i \alpha)=\mathcal{C}_{\epsilon_{0}}(\mathcal{H}, i \beta)$ is a connected component of $\Lambda_{\epsilon_{0}}^{\mathrm{Ham}}(\mathcal{H}, \mathbb{F})$. Since $\mathcal{C}_{\epsilon_{0}}(\mathcal{H}, i \alpha) \cap$ $\Lambda(\mathcal{H})=\{i \alpha, i \beta\}$, by Theorem 5.9 the component $\mathcal{C}_{\epsilon_{0}}(\mathcal{H}, i \alpha)$ has positive sign characteristic. Hence by Corollary 5.10 , we have $\mathcal{C}_{\epsilon_{0}}(\mathcal{H}, i \alpha) \subset i \mathbb{R}$.

Note that the map $\epsilon \mapsto \Lambda_{\epsilon}^{\mathrm{Ham}}(\mathcal{H}, \mathbb{F})$ is continuous and monotonically increasing and that the components of $\Lambda_{\epsilon}^{\mathrm{Ham}}(\mathcal{H}, \mathbb{F})$ are closed connected sets. Hence there is a $\delta_{0}>0$ such that the component $\mathcal{C}_{\epsilon}(\mathcal{H}, i \alpha)$ remains disjoint from the rest of the components of $\Lambda_{\epsilon}^{\mathrm{Ham}}(\mathcal{H}, \mathbb{F})$ for all $\epsilon_{0} \leq \epsilon<\epsilon_{0}+\delta_{0}$. This shows that $\mathcal{C}_{\epsilon_{0}+\delta}(\mathcal{H}, i \alpha) \cap \Lambda(\mathcal{H})=\{i \alpha, i \beta\}$ for all $0 \leq \delta<\delta_{0}$. Consequently, by Theorem $5.9, \mathcal{C}_{\epsilon_{0}+\delta}(\mathcal{H}, i \alpha)$ has positive sign characteristic and hence $\mathcal{C}_{\epsilon_{0}+\delta}(\mathcal{H}, i \alpha) \subset i \mathbb{R}$ for all $0 \leq \delta<\delta_{0}$. This completes the proof.

Observe that the assumptions in Theorem 5.11 make sure that components of the Hamiltonian pseudospectrum $\Lambda_{\epsilon}^{\mathrm{Ham}}(\mathcal{H}, \mathbb{F})$ do not coalesce at a point $i \omega$, for some $\omega \in[\alpha, \beta] \backslash\left\{\omega_{0}\right\}$, for all $\epsilon<\epsilon_{0}$.

We now consider the special case when all eigenvalues of a Hamiltonian matrix $\mathcal{H}$ are purely imaginary and each eigenvalue has either positive or negative sign character-

Copyright @ ( by SIAM. Unauthorized reproduction of this article is prohibited. 
istic. Then by Theorem 5.11 we conclude that a purely imaginary eigenvalue of $\mathcal{H}$ can be moved off of the imaginary axis only after the eigenvalue is made to coalesce with an imaginary eigenvalue of $\mathcal{H}$ of opposite sign characteristic. In order to analyze this issue further, we proceed as follows.

Let $\mathcal{H} \in \mathbb{F}^{2 n, 2 n}$ be a Hamiltonian matrix whose eigenvalues are all purely imaginary, and define

$$
\begin{aligned}
\rho_{\mathbb{F}}(\mathcal{H}):=\inf \left\{\|\mathcal{E}\|_{2}: \mathcal{E} \in \mathbb{F}^{2 n, 2 n}, \quad(\mathcal{J E})^{\star}=\mathcal{J E},\right. \\
\mathcal{H}+\mathcal{E} \text { has a nonimaginary eigenvalue }\}, \\
R_{\mathbb{F}}(\mathcal{H}):=\inf \left\{\|\mathcal{E}\|_{2}: \mathcal{E} \in \mathbb{F}^{2 n, 2 n}, \quad(\mathcal{J E})^{\star}=\mathcal{J E},\right. \\
\mathcal{H}+\mathcal{E} \text { has a } \mathcal{J} \text {-neutral eigenvector }\}
\end{aligned}
$$

Obviously, $\rho_{\mathbb{F}}(\mathcal{H}) \geq R_{\mathbb{F}}(\mathcal{H})$. The following result shows equality and how to compute either using the singular value function $\omega \mapsto \sigma_{\min }(\mathcal{H}-i \omega I), \omega \in \mathbb{R}$.

THEOREM 5.12. Let $\mathcal{H} \in \mathbb{F}^{2 n, 2 n}$ be a Hamiltonian matrix whose eigenvalues are all purely imaginary, and let $f(\omega)=\sigma_{\min }(\mathcal{H}-i \omega I), \omega \in \mathbb{R}$. Then the following assertions hold.

(i) If at least one eigenvalue of $\mathcal{H}$ has mixed sign characteristic, then $R_{\mathbb{F}}(\mathcal{H})=$ $\rho_{\mathbb{F}}(\mathcal{H})=0$.

(ii) Suppose that each eigenvalue of $\mathcal{H}$ has either positive or negative sign characteristic. Let $i \mathcal{I}_{1}, \ldots, i \mathcal{I}_{q} \subset i \mathbb{R}$ denote the closed intervals on the imaginary axis whose end points are adjacent eigenvalues of $\mathcal{H}$ with opposite sign characteristics. Then we have

$$
R_{\mathbb{F}}(\mathcal{H})=\rho_{\mathbb{F}}(\mathcal{H})=\min _{1 \leq k \leq q} \max _{\omega \in \mathcal{I}_{k}} f(\omega)
$$

Consider an interval $\mathcal{I} \in\left\{\mathcal{I}_{1}, \ldots, \mathcal{I}_{q}\right\}$ satisfying

$$
\min _{1 \leq k \leq q} \max _{\omega \in \mathcal{I}_{k}} f(\omega)=\max _{\omega \in \mathcal{I}} f(\omega)=f\left(\omega_{0}\right), \quad \omega_{0} \in \mathcal{I} .
$$

Suppose that iI is given by $i \mathcal{I}=[i \alpha, i \beta]$. Then the claims (a) and (b) of Theorem 5.11 hold. For the $\mathcal{J}$-neutral unit vector $v_{0}$ of claim (b) in Theorem 5.11 , consider the matrices

$$
\begin{aligned}
& \mathcal{E}^{0}:=\lambda_{\min }\left(\omega_{0}\right) \mathcal{J} v_{0} v_{0}^{\star}, \\
& \mathcal{K}^{0}:=\lambda_{\min }\left(\omega_{0}\right) \mathcal{J}\left[\begin{array}{ll}
v_{0} & \overline{v_{0}}
\end{array}\right]\left[\begin{array}{ll}
v_{0} & \bar{v}_{0}
\end{array}\right]^{+}, \\
& \mathcal{E}_{\mu}:=\mathcal{G}\left(v_{0}, \mu v_{0}\right), \\
& \mathcal{K}_{\mu}:=\mathcal{G}\left(\left[\begin{array}{ll}
v_{0} & \bar{v}_{0}
\end{array}\right],\left[\begin{array}{ll}
\mu v_{0} & \mu \bar{v}_{0}
\end{array}\right]\right), \quad \mu \in \mathbb{C},
\end{aligned}
$$

where $\mathcal{G}(\cdot, \cdot)$ is defined as in Theorem 3.2. Then $\mathcal{E}^{0}$ is Hamiltonian, $\mathcal{K}^{0}$ is real and Hamiltonian, $\left(\mathcal{H}+\mathcal{E}^{0}\right) v_{0}=\left(\mathcal{H}+\mathcal{K}^{0}\right) v_{0}=i \omega_{0} v_{0}$, and $\left\|\mathcal{E}^{0}\right\|_{2}=\left\|\mathcal{K}^{0}\right\|_{2}=$ $f\left(\omega_{0}\right)$. For any $\mu \in \mathbb{C}$ the matrix $\mathcal{E}_{\mu}$ is Hamiltonian, and $\left(\mathcal{H}+\mathcal{E}^{0}+\mathcal{E}_{\mu}\right) v_{0}=$ $\left(i \omega_{0}+\mu\right) v_{0}$. If $\omega_{0}=0$ and $\mathcal{H}$ is real, then $v_{0}$ can be chosen as a real vector. Then $\mathcal{E}^{0}+\mathcal{E}_{\mu}$ is a real matrix for all $\mu \in \mathbb{R}$. If $\omega_{0} \neq 0$ and $\mathcal{H}$ is real, then for any $\mu \in \mathbb{C}, \mathcal{K}_{\mu}$ is a real Hamiltonian matrix satisfying $\left(\mathcal{H}+\mathcal{K}^{0}+\right.$ $\left.\mathcal{K}_{\mu}\right) v_{0}=\left(i \omega_{0}+\mu\right) v_{0}$.

Copyright ( $)$ by SIAM. Unauthorized reproduction of this article is prohibited. 
Proof. Part (i) is obvious.

For part (ii), let $v$ denote the right-hand side of (5.8), let $\omega_{k} \in \mathcal{I}_{k}$ be such that $f\left(\omega_{k}\right)=\max _{\omega \in \mathcal{I}_{k}} f(\omega)$, and let the numbering be such that $\omega_{1}<\omega_{2}<\cdots<\omega_{q}$. Then for $0 \leq \epsilon<v$ and all $k$ we have $\epsilon<f\left(\omega_{k}\right)$, and hence $\Lambda_{\epsilon}^{\text {Ham }}(\mathcal{H}, \mathbb{F}) \cap$ $\left\{i \omega_{1}, \ldots, i \omega_{q}\right\}=\varnothing$. Furthermore, by the definition of the intervals $\mathcal{I}_{k}$, the numbers $i \omega_{k}$ separate the eigenvalues of $\mathcal{H}$ of different sign characteristic. More precisely, for any $k$, all eigenvalues of $\mathcal{H}$ that are contained in the interval $i\left(\omega_{k-1}, \omega_{k}\right) \subset i \mathbb{R}$ have the same sign characteristic (here we use the notation $\omega_{0}=-\infty, \omega_{q+1}=\infty$ ). Let $\mathcal{H}(t)=\mathcal{H}+t \mathcal{E}$, where $t \in \mathbb{R}$ and $\mathcal{E}$ is Hamiltonian with $\|\mathcal{E}\|_{2} \leq \epsilon$. Furthermore, let $t_{0}=\sup \{\theta \in[0,1] \mid \Lambda(\mathcal{H}(t)) \subset i \mathbb{R}$ for all $t \in[0, \theta]\}$, and let $\Lambda_{0}=\bigcup_{t \in\left[0, t_{0}\right]} \Lambda(\mathcal{H}(t))$. Suppose that $t_{0}<1$. Then by Theorem 4.3 the matrix $\mathcal{H}\left(t_{0}\right)$ has a $\mathcal{J}$-neutral eigenvector. However, we have $\Lambda_{0} \subseteq \Lambda_{\epsilon}^{\operatorname{Ham}}(\mathcal{H}, \mathbb{F})$ and hence $\Lambda_{0} \cap\left\{i \omega_{1}, \ldots, i \omega_{q}\right\}=\varnothing$. Thus each connected component $\mathcal{C} \subset i \mathbb{R}$ of $\Lambda_{0}$ does not contain eigenvalues of $\mathcal{H}=\mathcal{H}(0)$ of opposite sign characteristic. Hence each connected component $\mathcal{C}$ of $\Lambda_{0}$ has either positive or negative sign characteristic. This contradicts the assumption that $\mathcal{H}\left(t_{0}\right)$ has a $\mathcal{J}$-neutral eigenvector. Thus $t_{0}=1$. It follows that $v \leq R_{\mathbb{F}}(\mathcal{H}), v \leq \rho_{\mathbb{E}}(\mathcal{H})$, and $\Lambda_{\epsilon}^{\operatorname{Ham}}(\mathcal{H}, \mathbb{F}) \subset i \mathbb{R}$ for all $\epsilon<v$. Furthermore, each connected component of $\Lambda_{\epsilon}^{\mathrm{Ham}}(\mathcal{H}, \mathbb{C}), \epsilon<v$, has either positive or negative sign characteristic.

Now, let $\omega_{0}$ and $\mathcal{I}$ be as in (5.9). Since $i \mathcal{I}=[i \alpha, i \beta]$ and the eigenvalues $i \alpha$ and $i \beta$ have opposite sign characteristic, the assumptions (i) and (ii) of Theorem 5.11 are automatically satisfied, and hence the assertions (a), (b), and (c) of Theorem 5.11 hold. The statements about the matrices $\mathcal{E}^{0}, \mathcal{E}_{\mu}, \mathcal{K}^{0}, \mathcal{K}_{\mu}$ imply that $R_{\mathbb{F}}(\mathcal{H}) \leq v$ and $\rho_{\mathbb{F}}(\mathcal{H}) \leq v$ which follows from Theorem 4.5 and Proposition 5.3.

Example 5.13. The eigenvalues $\pm 10 i$ of the matrix $\mathcal{H}_{1}$ from Example 5.5 have mixed sign characteristics. Thus $R_{\mathbb{F}}\left(\mathcal{H}_{1}\right)=\rho_{\mathbb{F}}\left(\mathcal{H}_{1}\right)=0$.

Example 5.14. Consider the Hamiltonian matrices

$$
\mathcal{H}_{3}=\left[\begin{array}{cccc}
0 & 0 & 1 & 0 \\
0 & 0 & 0 & 1 \\
-1 & 0 & 0 & 0 \\
0 & -4 & 0 & 0
\end{array}\right], \quad \mathcal{H}_{4}=\left[\begin{array}{cccc}
0 & 0 & 1 & 0 \\
0 & 0 & 0 & -1 \\
-1 & 0 & 0 & 0 \\
0 & 4 & 0 & 0
\end{array}\right]
$$

Both matrices have the same spectrum $\Lambda\left(\mathcal{H}_{k}\right)=\{ \pm i, \pm 2 i\}, k=3,4$, and their eigenvalue curves $\omega \mapsto \lambda_{j}\left(\mathcal{J}\left(\mathcal{H}_{k}-i \omega I\right)\right)$ and singular value curves $\omega \mapsto \sigma_{j}\left(\mathcal{H}_{k}-i \omega I\right)$ are depicted in Figure 5.2.

Here the singular value curves for $\mathcal{H}_{3}$ and $\mathcal{H}_{4}$ coincide, and the graphs of the functions $\omega \mapsto \sigma_{\min }\left(\mathcal{H}_{k}-i \omega I\right)$ and $\omega \mapsto \lambda_{\min }\left(\mathcal{H}_{k}-i \omega I\right)$ are depicted as thick curves. From the slopes of the $\lambda_{j}$-curves at their crossing points with the real axis we can again read off the sign characteristics of the eigenvalues $\pm i, \pm 2 i$, and we see that for the matrix $\mathcal{H}_{3}$ the eigenvalues $-2 i$ and $-i$ have negative sign characteristic, while the eigenvalues $i$ and $2 i$ have positive sign characteristic. Thus the only pair of adjacent eigenvalues of $\mathcal{H}_{3}$ with opposite sign characteristic is $(-i, i)$. The maximum of the function $f(\omega)=\sigma_{\min }\left(\mathcal{H}_{3}-\right.$ $i \omega I)$ in the corresponding interval $[-1,1]$ is 1 . Thus $R_{\mathbb{F}}\left(\mathcal{H}_{3}\right)=\rho_{\mathbb{F}}\left(\mathcal{H}_{3}\right)=1$.

For the matrix $\mathcal{H}_{4}$ the eigenvalues $-2 i$ and $i$ have positive sign characteristic, while the eigenvalues $-i$ and $2 i$ have negative sign characteristic. The pairs of adjacent eigenvalues of $\mathcal{H}_{4}$ of opposite sign characteristic are $(-2 i,-i),(-i, i),(i, 2 i)$, and the maxima of the function $f(\omega)=\sigma_{\min }\left(\mathcal{H}_{3}-i \omega I\right)$ in the corresponding intervals $[-2,-1],[-1,1],[1,2]$ are $v, 1, v$, respectively, where $v \approx 0.43$. Thus $R_{\mathbb{F}}\left(\mathcal{H}_{4}\right)=$ $\rho_{\mathbb{F}}\left(\mathcal{H}_{4}\right)=v$.

Copyright ( $\odot$ by SIAM. Unauthorized reproduction of this article is prohibited. 

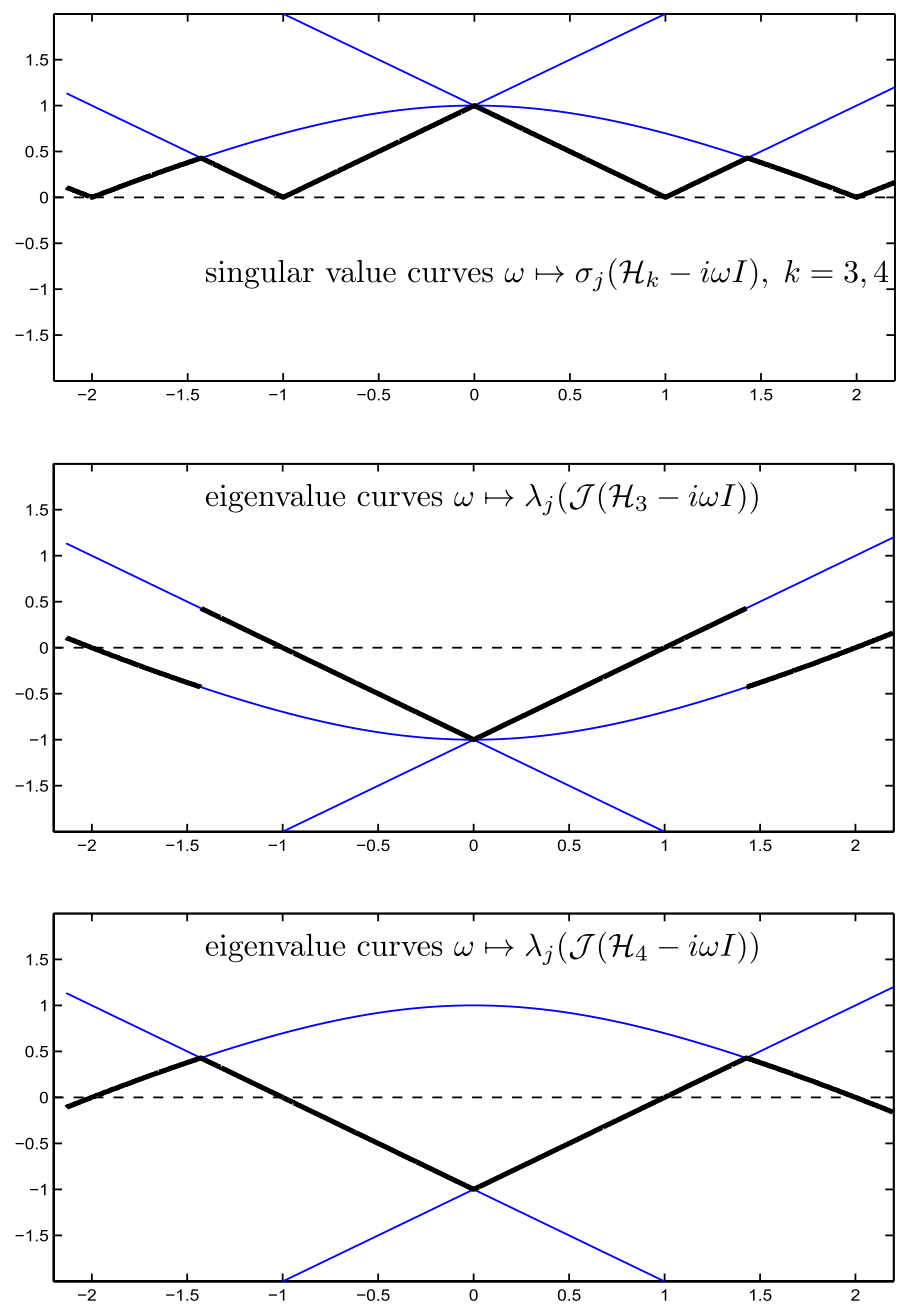

FIG. 5.2. Eigenvalue and singular value curves for Example 5.14.

In this section we have discussed the process of constructing the perturbations that move the eigenvalues off the imaginary axis. These will be used in the algorithm of the next section.

6. An algorithm to compute a bound for the distance to boundedrealness. In this section we discuss a numerical method to approximately solve Problems A and B, i.e., to compute an upper bound for the smallest perturbation that moves all eigenvalues of a Hamiltonian matrix off the imaginary axis or outside a strip $S_{\tau}$ parallel to the imaginary axis. We cover both Problems A and B by different choices of $\tau$; i.e., Problem A is the case when $\tau=0$.

In general it is an open problem to analytically classify the smallest perturbation that solves these two problems. Instead, we determine an upper bound for the smallest perturbation by solving small problems of size $2 \times 2$ or $4 \times 4$ in the real case. We also discuss only the special case that the Hamiltonian matrix has only purely imaginary eigenvalues. Numerically we can restrict ourselves to the latter case because we can first 
use the methods in [7], [27] to compute a partial Hamiltonian Schur form of the matrix $\mathcal{H}$ as in (1.2); i.e., we determine an orthogonal (unitary) and symplectic matrix $Q_{0}$ such that for the transformed Hamiltonian matrix

$$
Q_{0}^{\star} \mathcal{H} Q_{0}=\left[\begin{array}{cc|rc}
F_{11} & F_{12} & G_{11} & G_{12} \\
0 & F_{22} & G_{21} & G_{22} \\
\hline 0 & 0 & -F_{11}^{\star} & 0 \\
0 & H_{22} & -F_{12}^{\star} & -F_{22}^{\star}
\end{array}\right],
$$

we have that $F_{11}$ is upper triangular in the complex case or quasi-upper triangular in the real case and contains those eigenvalues of $\mathcal{H}$ which lie (within the perturbation analysis of Hamiltonian matrices [28]) outside of the strip $S_{\tau}=\{z \in \mathbb{C} \mid-\tau<\mathfrak{R} z<\tau\}$.

By restricting the perturbations to the Hamiltonian submatrix

$$
\tilde{H}_{2}=\left[\begin{array}{cc}
F_{22} & G_{22} \\
H_{22} & -F_{22}^{\star}
\end{array}\right]
$$

which contains all the eigenvalues that lie within the strip $S_{\tau}$, we determine an upper bound for the smallest perturbation to the full matrix. A reason why it may not be the smallest perturbation is that it may be possible that the smallest perturbation moves two eigenvalues of $F_{11}$ that lie outside the strip $S_{\tau}$ into $S_{\tau}$ and then combines them with other eigenvalues in $S_{\tau}$ to get the globally smallest perturbation; see [28]. But since we are treating eigenvalues in badges of two or four at a time, there may be a more global small perturbation that moves all the eigenvalues together at the same time.

There are several possibilities for the parameter $\tau$ that describes the width of the strip $S_{\tau}$. It can either be preassigned to achieve a robust bounded-realness margin, or if we want only to make sure that the eigenvalues are robustly off the imaginary axis, within the usual round-off error analysis, then, since an $O(\epsilon)$ perturbation to a $2 \times 2$ Jordan block can produce an $O\left(\epsilon^{1 / 2}\right)$ change in the eigenvalue, it seems reasonable to choose $\tau=O\left(u^{1 / 2}\right)$, where $u$ is the round-off unit. If there is reason to think that some of the nonimaginary eigenvalues close to the imaginary axis are the effect of round-off errors on a $k \times k$ Jordan block, then one should choose $\tau=O\left(u^{1 / k}\right)$.

Since, due to round-off errors, we cannot be sure whether eigenvalues of $\tilde{H}_{2}$ are on or off the imaginary axis, in view of the discussed perturbation analysis we first regularize the problem by perturbing $\tilde{H}_{2}$ to $H_{2}=\tilde{H}_{2}+\Delta H_{2}$ with

$$
\Delta H_{2}=\left[\begin{array}{c|c}
\Delta F_{22} & \Delta G_{22} \\
\hline \Delta H_{22} & -\Delta F_{22}^{\star}
\end{array}\right]
$$

so that all eigenvalues of $H_{2}=\tilde{H}_{2}+\Delta H_{2}$ are on the imaginary axis. This can be done by reversing the perturbations that we have introduced in Proposition 5.3.

In this way the following approach, which combines nearest purely imaginary eigenvalues of opposite sign, is not restricted, and we do not have to make a preliminary decision as to which eigenvalues are purely imaginary and which are not.

For each eigenvalue pair that the partial Hamiltonian Schur form produces outside the imaginary axis, a minimal perturbation $\mathcal{E}_{2}$ that performs this task is given by Proposition 5.3. In the following we recursively work on the matrix $\mathcal{H}_{2}$ and perturb one pair of purely imaginary eigenvalues at a time. Again this may have the effect of increasing the bound for the minimal perturbation since there may be a smaller perturbation that moves several pairs at the same time.

Copyright () by SIAM. Unauthorized reproduction of this article is prohibited. 
For each chosen pair of purely imaginary eigenvalues with opposite sign characteristic (which pair of purely imaginary eigenvalues is to be chosen is discussed below) we first compute the smallest perturbation that leads to a coalescence of the pseudospectral components as described in Theorem 5.12. In this way we produce an eigenvalue of mixed sign characteristic at a point $i \gamma$. If we want to solve Problem A, then this perturbation is sufficient. If we want to solve Problem B, then we move this pair of eigenvalues to the pair $\pm \tau+i \gamma$ on the boundary of $S_{\tau}$. In both cases we save the perturbation $\mathcal{E}_{2}$. By taking a direct sum with an appropriate 0 matrix we generate a perturbation $\mathcal{E}$ to the matrix $\mathcal{H}$ as well as its norm $\delta$. Since in both cases the perturbed eigenvalue belongs to the part where a Hamiltonian Schur form exists, we can deflate this eigenvalue pair from $\mathcal{H}_{2}$ and continue with a smaller problem $\mathcal{H}_{2}$ for which we proceed as before.

Algorithm 1.

Input: A Hamiltonian matrix $\mathcal{H} \in \mathbb{F}^{2 n, 2 n}$ that has only purely imaginary eigenvalues and a value $\tau>0$ for the width of the strip $S_{\tau}$ around the imaginary axis.

Output: A Hamiltonian matrix $\mathcal{E} \in \mathbb{F}^{2 n, 2 n}$ such that at least one pair (quadruple in the real case) of eigenvalues of $\mathcal{H}+\mathcal{E}$ is outside the open strip $S_{\tau}$.

Step 1: Compute the eigenvalues $i \alpha_{k}, \alpha_{k} \in \mathbb{R}, k=1, \ldots, 2 n$, and associated eigenvectors $v_{k} \in \mathbb{C}^{2 n}$ of $\mathcal{H}$. By using the reordering of the Hamiltonian Schur form [5], order the eigenvalues such that the eigenvalues arise in the order $\alpha_{k} \leq \alpha_{k+1}$ on the diagonal. Then compute the eigenvectors. (For multiple eigenvalues, consider the invariant subspace spanned by the columns of a matrix $V$ associated with this eigenvalue.)

Step 2: Compute the sign characteristics of the eigenvalues (i.e., the signs of $i v_{k}^{\star} \mathcal{J} v_{k}$, $k=1, \ldots, 2 n$, or the inertia of the matrix $i V^{\star} \mathcal{J} V$ in the case of multiple eigenvalues).

Step 3: If there is a multiple eigenvalue of mixed sign characteristic (i.e., $\alpha_{k}=\alpha_{k+1}$ ) and $\operatorname{sign}\left(i v_{k}^{\star} \mathcal{J} v_{k}\right) \operatorname{sign}\left(i v_{k+1}^{\star} \mathcal{J} v_{k+1}\right)<0$, then let $v_{-}:=v_{k}, v_{+}:=v_{k+1}$, and go to Step 6.

Step 4: For each pair of adjacent eigenvalues $i \alpha_{k}, i \alpha_{k+1}$ with opposite sign characteristic compute the maximum $m_{k}:=\max _{\omega \in\left[\alpha_{k}, \alpha_{k+1}\right]} f(\omega)$, where $f(\omega)$ $=\sigma_{\min }(\mathcal{H}-i \omega I)=\left|\lambda_{\min }(\mathcal{J}(\mathcal{H}-i \omega I))\right|, \omega \in \mathbb{R}$.

Remark. Since $f$ satisfies $|f(\omega)-f(\tilde{\omega})| \leq|\omega-\tilde{\omega}|$, the maxima can be found by evaluating $f$ on a coarse grid.

Step 5: From the eigenvalues found in Step 4 select an eigenvalue $i \alpha_{k_{0}}$ such that $m_{k_{0}}=\min m_{k}$. By Theorems 5.11 and 5.12 there is an $\omega_{0} \in\left[\alpha_{k_{0}}, \alpha_{k_{0}+1}\right]$ such that the function $f$ is strictly increasing in $\left[\alpha_{k_{0}}, \omega_{0}\right]$ and strictly decreasing in $\left[\omega_{0}, \alpha_{k_{0}+1}\right]$ (hence $f\left(\omega_{0}\right)=m_{k_{0}}$ ). By using a trisection method, determine a small interval $\left[\omega_{-}, \omega_{+}\right]$that contains $\omega_{0}$. Let $v_{ \pm}$be eigenvectors to the eigenvalues $\lambda_{\min }\left(\mathcal{J}\left(\mathcal{H}-i \omega_{ \pm} I\right)\right)$. The real numbers $-i v_{ \pm}^{\star} \mathcal{J} v_{ \pm}$are the slopes of the curve $\omega \mapsto \lambda_{\min }(\omega):=\lambda_{\min }(\mathcal{J}(\mathcal{H}-i \omega I))$ at $\omega=\omega_{ \pm}$. Again by Theorems 5.11 and 5.12 either $f(\omega)=\lambda_{\min }(\omega)$ for all $\omega \in\left[\alpha_{k_{0}}, \alpha_{k_{0}+1}\right]$ or $f(\omega)=-\lambda_{\min }(\omega)$ for all $\omega \in\left[\alpha_{k_{0}}, \alpha_{k_{0}+1}\right]$. Thus $\operatorname{sign}\left(i v_{+}^{\star} \mathcal{J} v_{+}\right) \operatorname{sign}\left(i v_{-}^{\star} \mathcal{J} v_{-}\right)<0$.

Step 6: Compute $t \in[0,1]$ such that $u_{t}^{\star} \mathcal{J} u_{t}=0$, where $u_{t}=t v_{+}+(1-t) v_{-}$, and let $v_{0}=u_{t} /\left\|u_{t}\right\|$. Then $v_{0}$ is an approximate $\mathcal{J}$-neutral eigenvector to the eigenvalue $\lambda_{\min }\left(\mathcal{J}\left(\mathcal{H}-i \omega_{0} I\right)\right)$.

Step 7: Let $\mu=\tau$.

Copyright $@$ ( by SIAM. Unauthorized reproduction of this article is prohibited. 
Step 8: Let $\tilde{\mathcal{E}}=\mathcal{E}^{0}+\mathcal{E}_{\mu}$ in the complex case, and $\tilde{\mathcal{E}}=\mathcal{K}^{0}+\mathcal{K}_{\mu}$ in the real case, where $\mathcal{E}^{0}, \mathcal{E}_{\mu}, \mathcal{K}^{0}$, and $\mathcal{K}_{\mu}$ are defined by (5.10). Then by Theorem $5.12, \mathcal{H}+\tilde{\mathcal{E}}$ has (approximately) the two eigenvalues $i \omega_{0} \pm \mu$ in the complex case and the four eigenvalues $\pm i \omega_{0} \pm \mu$ in the real case. Due to rounding errors $\tilde{\mathcal{E}}$ may have a slight departure from being Hamiltonian. A Hamiltonian matrix close to $\tilde{\mathcal{E}}$ is $\mathcal{E}=-\frac{1}{2} \mathcal{J}\left(\mathcal{J} \tilde{\mathcal{E}}+(\mathcal{J} \tilde{\mathcal{E}})^{\top}\right)$.

Step 9: Check whether at least two eigenvalues of $\mathcal{H}+\mathcal{E}$ are outside the strip $S_{\tau}$. If this is not the case, increase $\mu$ and return to Step 8.

Applying this algorithm recursively we obtain (as a sum of all the single perturbation matrices) a perturbation matrix $\Delta \mathcal{H}$ such that, at least in theory, all eigenvalues of the perturbed Hamiltonian matrix $\mathcal{H} \leftarrow H+\Delta \mathcal{H}$ lie outside the strip $S_{\tau}$. Due to round-off errors in the computations, however, it may happen that some eigenvalues of $\mathcal{H}$ have moved back towards the imaginary axis. Therefore, as in Step 9, it is advisable to check the spectrum of $\mathcal{H}$ to see whether the eigenvalues are safely removed from the imaginary axis in the sense that a Hamiltonian perturbation up to the size of the round-off error cannot move the eigenvalues back to the imaginary axis.

So, suppose that $\mathcal{H}$ is the Hamiltonian matrix obtained by a successive application of Algorithm 1 until all eigenvalues have been moved off the imaginary axis. Then for a given tolerance $\tau$ we would like to test that the eigenvalues of $\mathcal{H}$ are robustly away from the imaginary axis in the sense that $\mathcal{H}+\mathcal{E}$ does not have an imaginary eigenvalue for any Hamiltonian perturbation $\mathcal{E}$ such that $\|\mathcal{E}\|_{2} \leq \tau$. Given a Hamiltonian matrix $\mathcal{H} \in \mathbb{F}^{2 n, 2 n}$, define

$$
\beta_{\mathbb{F}}(\mathcal{H}):=\min \left\{\|\mathcal{E}\|_{2}: \mathcal{E} \in \mathbb{F}^{2 n, 2 n},(\mathcal{J E})^{\star}=\mathcal{J E} \text {, and } \Lambda(\mathcal{H}+\mathcal{E}) \cap i \mathbb{R} \neq \varnothing\right\} .
$$

Then $\beta_{\mathbb{F}}(\mathcal{H})$ is the distance from $\mathcal{H}$ to the Hamiltonian matrices having a purely imaginary eigenvalue. Moreover, it follows from Corollary 5.7 that $\beta_{\mathbb{F}}(\mathcal{H})=$ $\min \left\{\epsilon: \Lambda_{\epsilon}^{\mathrm{Ham}}(\mathcal{H}, \mathbb{F}) \cap i \mathbb{R} \neq \varnothing\right\}=\Lambda_{\epsilon}(\mathcal{H}, \mathbb{C}) \cap i \mathbb{R} \neq \varnothing$. This shows that $\beta_{\mathbb{F}}(\mathcal{H})$ is the same for $\mathbb{F}=\mathbb{R}$ and $\mathbb{F}=\mathbb{C}$ and that it can be read off from the unstructured pseudospectrum $\Lambda_{\epsilon}(\mathcal{H}, \mathbb{C})$ of $\mathcal{H}$.

For the Hamiltonian matrix $\mathcal{H}$ computed by this procedure, we need to test whether or not $\beta_{\mathbb{F}}(\mathcal{H})>\tau$. This can be done by computing the Hamiltonian pseudospectrum $\Lambda_{\tau}(\mathcal{H}, \mathbb{C})$ with the method of [19] and testing whether or not $\Lambda_{\tau}(\mathcal{H}, \mathbb{C}) \cap i \mathbb{R}=\varnothing$. Alternatively, we compute the eigenvalues of $\mathcal{H}-\tau \mathcal{J}$ and $\mathcal{H}+\tau \mathcal{J}$. If these matrices do not have a purely imaginary eigenvalue, then by Theorem 2 of [6] we have $\beta_{\mathbb{F}}(\mathcal{H})>\tau$, and hence the eigenvalues of $\mathcal{H}$ are robustly away from the imaginary axis.

The computational costs of Algorithm 1 can be significantly reduced by modifying the choice of the nearest purely imaginary eigenvalues that are brought to coalescence using the following idea which may, however, in some rare cases, lead to a larger perturbation than necessary. To choose the pair $\left(i \gamma_{1}, i \gamma_{2}\right)$ or in the real case a quadruple $\left(i \gamma_{1},-i \gamma_{1}, i \gamma_{2},-i \gamma_{2}\right)$ of purely imaginary eigenvalues that are moved together at a point $\pm \tau+i \gamma$, we may proceed as follows. Assuming that the eigenvalues of $\mathcal{H}$ are all simple, we choose a pair of purely imaginary eigenvalues $\left(i \gamma_{j}, i \gamma_{l}\right)$ of opposite sign characteristic for which the ratio

$$
\frac{\left|\gamma_{j}-\gamma_{l}\right|}{\kappa\left(\gamma_{j}\right)+\kappa\left(\gamma_{l}\right)}
$$

is the smallest among all such pairs, where $\kappa\left(\gamma_{j}\right)$ is the condition number of the eigenvalue $i \gamma_{j}$. We arrive at this choice from the first order perturbation analysis of the 
eigenvalues. Indeed, by first order perturbation of eigenvalues, it follows that the components of $\Lambda_{\epsilon}^{\operatorname{Ham}}(\mathcal{H}, \mathbb{F})$ containing $i \gamma_{j}$ and $i \gamma_{l}$ are approximately the intervals $i\left[\gamma_{j}-\kappa\left(\lambda_{j}\right) \epsilon, \gamma_{j}+\kappa\left(\gamma_{j}\right) \epsilon\right]$ and $i\left[\gamma_{l}-\kappa\left(\lambda_{l}\right) \epsilon, \gamma_{l}+\kappa\left(\gamma_{l}\right) \epsilon\right]$, respectively, for all small $\epsilon$. Therefore, if the ratio (6.1) is the smallest, as $\epsilon$ increases gradually these two components are likely to coalesce before the other components.

6.1. A numerical example. To illustrate our procedure, we apply Algorithm 1 to the matrix

$$
\mathcal{H}=\left[\begin{array}{cccccccc}
-73 & -86 & 54 & -99 & 93 & -58 & 80 & 77 \\
1 & -4 & 59 & 54 & -58 & -61 & 4 & 1 \\
-24 & -31 & -4 & -86 & 80 & 4 & 27 & 26 \\
-26 & -24 & 1 & -73 & 77 & 1 & 26 & 24 \\
-24 & -26 & -1 & -77 & 73 & -1 & 24 & 26 \\
-26 & -27 & -4 & -80 & 86 & 4 & 31 & 24 \\
-1 & -4 & 61 & 58 & -54 & -59 & 4 & -1 \\
-77 & -80 & 58 & -93 & 99 & -54 & 86 & 73
\end{array}\right]
$$

The matrix $\mathcal{H}$ has the purely imaginary spectrum

$$
\Lambda(\mathcal{H})=\{ \pm 4 i, \pm 10 i, \pm 16 i, \pm 18 i\}
$$

The intervals bounded by adjacent eigenvalues with opposite sign characteristic are $i \mathcal{I}_{1}=[-16 i,-10 i], i \mathcal{I}_{2}=[-10 i,-4 i], i \mathcal{I}_{3}=[-4 i, 4 i], i \mathcal{I}_{4}=[4 i, 10 i]$, and $i \mathcal{I}_{5}=$ $[10 i, 16 i]$.

Algorithm 1 computes the maximum of the function $\omega \mapsto f(\omega)=\left|\lambda_{\min }(\mathcal{H}-i \omega I)\right|$ in each of the intervals $\mathcal{I}_{k}$. The minimum of these maxima is attained in the interval $\mathcal{I}_{1}$ at $\omega_{0} \approx-13.9356$. A corresponding normalized $\mathcal{J}$-neutral eigenvector (see Step 6 ) is

$$
v_{0}=\left[\begin{array}{c}
0.5854-0.2940 i \\
-0.1559-0.1188 i \\
-0.1238-0.0445 i \\
-0.1145-0.0459 i \\
-0.1081-0.0593 i \\
-0.1130-0.0673 i \\
-0.1907-0.0449 i \\
-0.5988-0.2655 i
\end{array}\right]
$$

For the width of the strip $S_{\tau}$ we choose $\tau=0.1$. Then the output of the algorithm is the matrix (for layout reasons displayed only with three digits)

$$
\mathcal{E}=10^{-2} *\left[\begin{array}{cccccccc}
5.74 & 3.38 & 0.81 & 0.02 & 2.46 & 2.68 & 0.81 & 4.30 \\
3.78 & 5.26 & -0.21 & -0.93 & 2.68 & 3.49 & -4.74 & 0.10 \\
0.61 & -3.70 & -2.21 & -1.40 & -0.81 & -4.74 & 7.39 & 5.17 \\
3.88 & -1.13 & -1.01 & -3.48 & 4.30 & 0.10 & 5.17 & 7.27 \\
-2.93 & -0.61 & -0.72 & 3.19 & -5.74 & -3.78 & -0.61 & -3.88 \\
-0.61 & -1.55 & -2.75 & 0.19 & -3.38 & -5.26 & 3.70 & 1.33 \\
-0.72 & -2.75 & 2.35 & 1.88 & -0.81 & 0.21 & 2.21 & 1.01 \\
3.19 & 0.19 & 1.88 & 1.88 & -0.02 & 0.93 & 1.40 & 3.48
\end{array}\right] .
$$

Copyright $@$ by SIAM. Unauthorized reproduction of this article is prohibited. 
The eigenvalues of $\mathcal{H}+\mathcal{E}$ are

$$
\Lambda(\mathcal{H}+\mathcal{E}) \approx\{0.1000 \pm 13.9356 i,-(0.1000 \pm 13.9356 i), \pm 17.6162 i, \pm 4.3627 i\}
$$

A Hamiltonian Schur decomposition of $\mathcal{H}+\mathcal{E}$ yields

$$
Q_{0}^{\star}(\mathcal{H}+\mathcal{E}) Q_{0}=\left[\begin{array}{cc|rc}
F_{11} & F_{12} & G_{11} & G_{12} \\
0 & F_{22} & G_{21} & G_{22} \\
\hline 0 & 0 & -F_{11}^{\star} & 0 \\
0 & H_{22} & -F_{12}^{\star} & -F_{22}^{\star}
\end{array}\right],
$$

where $Q_{0}$ is symplectic and orthogonal, and

$$
\begin{aligned}
F_{22} & =\left[\begin{array}{cc}
7.7958 & -5.9178 \\
7.3945 & -3.3404
\end{array}\right], \quad G_{22}=\left[\begin{array}{cc}
-30.8492 & -2.5331 \\
-2.5331 & 0.8874
\end{array}\right], \\
H_{22} & =\left[\begin{array}{ll}
11.0658 & -5.5371 \\
-5.5371 & -0.5170
\end{array}\right] .
\end{aligned}
$$

These blocks correspond to the purely imaginary eigenvalues of $\mathcal{H}+\mathcal{E}$. By applying Algorithm 1 again to the matrix

$$
\tilde{\mathcal{H}}=\left[\begin{array}{c|c}
F_{22} & G_{22} \\
\hline H_{22} & -F_{22}^{\star}
\end{array}\right]
$$

we obtain the output

$$
\tilde{\mathcal{E}}=\left[\begin{array}{cccc}
0.0707 & 1.2227 & 0.7015 & 0.0862 \\
1.2227 & 0.0306 & 0.0862 & 0.6986 \\
-2.1346 & 0.0862 & -0.0707 & -1.2227 \\
0.0862 & -2.1375 & -1.2227 & -0.0306
\end{array}\right]
$$

The computed eigenvalues of $\tilde{\mathcal{H}}+\tilde{\mathcal{E}}$ are

$$
\Lambda(\tilde{\mathcal{H}}+\tilde{\mathcal{E}})=\{0.1000 \pm 10.7368 i,-(0.1000 \pm 10.7368 i)\}
$$

Thus all eigenvalues of $\tilde{\mathcal{H}}+\tilde{\mathcal{E}}$ are outside the open strip $S_{\tau}$. Hence there is a real Hamiltonian matrix $\Delta \mathcal{H}$ with norm $\|\Delta \mathcal{H}\|_{2} \leq\|\mathcal{E}\|_{2}+\|\tilde{\mathcal{E}}\|_{2} \approx 3.005$ such that all eigenvalues of $\mathcal{H}+\Delta \mathcal{H}$ are outside the strip $S_{\tau}$.

7. Conclusion. We have presented a detailed perturbation analysis for eigenvalues of Hamiltonian matrices and discussed the construction of structured perturbations to Hamiltonian matrices that move eigenvalues off the imaginary axis and thereby discussed the computation of upper bounds for the distance to (robust) bounded-realness. The application of this new approach in the context of passivation problems will be discussed in a forthcoming work.

Copyright (C) by SIAM. Unauthorized reproduction of this article is prohibited. 
Acknowledgments. We thank two anonymous referees for their careful reading of the paper and for their suggestions which significantly improved the readability of the paper.

\section{REFERENCES}

[1] B. Adhikari, Backward Perturbation and Sensitivity Analysis of Structured Polynomial Eigenvalue Problems, Ph.D. thesis, Department of Mathematics, IIT Guwahati, Guwahati, India, 2008.

[2] B. AdhikARI And R. Alam, Structured mapping problems for a class of linearly structured matrices, Linear Algebra Appl., to appear.

[3] R. Alam and S. Bora, Effect of linear perturbation on spectra of matrices, Linear Algebra Appl., 368 (2003), pp. 329-342.

[4] A. C. Antoulas, Approximation of Large-Scale Dynamical Systems, SIAM, Philadelphia, 2005.

[5] R. Byers, A Hamiltonian QR algorithm, SIAM J. Sci. Statist. Comput., 7 (1986), pp. 212-229.

[6] R. Byers, A bisection method for measuring the distance of a stable matrix to the unstable matrices, SIAM J. Sci. Statist. Comput., 9 (1988), pp. 875-881.

[7] D. Chu, X. Liu, and V. Mehrmann, A numerical method for computing the Hamiltonian Schur form, Numer. Math., 105 (2007), pp. 375-412.

[8] C. P. Coelho, J. R. Phillips, and L. M. Silveira, Robust rational function approximation algorithm for model generation, in Proceedings of the 36th ACM/IEEE Design Automation Conference (DAC), New Orleans, LA, 1999, pp. 207-212.

[9] G. Freiling, V. Mehrmann, and H. Xu, Existence, uniqueness and parametrization of Lagrangian invariant subspaces, SIAM J. Matrix Anal. Appl., 23 (2002), pp. 1045-1069.

[10] R. W. Freund and F. JaRRe, An extension of the positive real lemma to descriptor systems, Optim. Methods Softw., 19 (2004), pp. 69-87.

[11] R. W. Freund, F. Jarre, and C. Vogelbusch, Nonlinear semidefinite programming: Sensitivity, convergence and an application in passive reduced-order modeling, Math. Program., 109 (2007), pp. 581-611.

[12] I. Gohberg, P. Lancaster, and L. Rodman, Indefinite Linear Algebra and Applications, Birkhäuser, Basel, 2005.

[13] G. H. Golub and C. F. Van Loan, Matrix Computations, 3rd ed., The Johns Hopkins University Press, Baltimore, 1996.

[14] S. GRIVEt-Talocia, Enforcing passivity of macromodels via spectral perturbation of Hamiltonian matrices, in Proceedings of the 7th IEEE Workshop on Signal Propagation on Interconnects, Siena, Italy, 2003, pp. 33-36.

[15] S. Grivet-Talocia, Passivity enforcement via perturbation of Hamiltonian matrices, IEEE Trans. Circuits Syst. I. Regul. Pap., 51 (2004), pp. 1755-1769.

[16] B. Gustavsen and A. Semlyen, Enforcing passivity for admittance matrices approximated by rational functions, IEEE Trans. Power Syst., 16 (2001), pp. 97-104.

[17] N. Ida And P. A. Bastos, Electromagnetics and Calculation of Fields, Springer-Verlag, New York, 1997.

[18] M. KaRow, $\mu$ values and spectral value sets for linear perturbation classes defined by a scalar product, SIAM J. Matrix Anal. Appl., to appear.

[19] M. Karow, E. Kokiopoulou, and D. Kressner, On the computation of structured singular values and pseudospectra, Systems Control Lett., 59 (2010), pp. 122-129.

[20] T. Kato, Perturbation Theory for Linear Operators, Springer-Verlag, New York, 1966.

[21] M. Konstantinov, V. Mehrmann, and P. Petkov, Perturbation analysis of Hamiltonian Schur and blockSchur forms, SIAM J. Matrix Anal. Appl., 23 (2001), pp. 387-424.

[22] P. Lancaster and L. Rodman, The Algebraic Riccati Equation, Oxford University Press, Oxford, 1995.

[23] P. Lancaster and M. Tismenetsky, The Theory of Matrices, 2nd ed., Academic Press, Orlando, FL, 1985.

[24] W.-W. Lin, V. Mehrmann, and H. Xu, Canonical forms for Hamiltonian and symplectic matrices and pencils Linear Algebra Appl., 302/303 (1999), pp. 469-533.

[25] C. MenL, Compatible Lie and Jordan Algebras and Applications to Structured Matrices and Pencils, Logos-Verlag, Berlin, 1999.

[26] V. Mehrmann, The Autonomous Linear Quadratic Control Problem, Theory and Numerical Solution, Lecture Notes in Control and Inform. Sci. 163, Springer-Verlag, Heidelberg, 1991.

[27] V. Mehrmann, C. SchröDer, and D. S. Watkins, A new block method for computing the Hamiltonian Schur form, Linear Algebra Appl., 431 (2009), pp. 350-368.

[28] V. Mehrmann and H. XU, Perturbation of purely imaginary eigenvalues of Hamiltonian matrices under structured perturbations, Electron. J. Linear Algebra, 17 (2008), pp. 234-257.

Copyright @ @ by SIAM. Unauthorized reproduction of this article is prohibited. 
[29] M. L. Overton and P. Van Dooren, On computing the complex passivity radius, in Proceedings of the 4th IEEE Conference on Decision and Control (ECC-CDC 2005), Seville, Spain, 2005, pp. 7960-7964.

[30] A. C. M. Ran and L. Rodman, Stability of invariant maximal semidefinite subspaces, Linear Algebra Appl., 62 (1984), pp. 51-86.

[31] A. C. M. Ran and L. Rodman, Stability of invariant Lagrangian subspaces I, Oper. Theory Adv. Appl. 32, I. Gohberg, ed., Birkhäuser, Basel, 1988, pp. 181-218.

[32] A. C. M. Ran and L. Rodman, Stability of invariant Lagrangian subspaces II, Oper. Theory Adv. Appl. 40, H. Dym, S. Goldberg, M. A. Kaashoek, and P. Lancaster, eds., Birkhäuser, Basel, 1989, pp. 391-425.

[33] F. Reluich, Perturbation Theory of Eigenvalue Problems, Gordon and Breach Science, New YorkLondon-Paris, 1969.

[34] D. Saraswat, R. Achar, AND M. NaKhIA, Enforcing passivity for rational function based macromodels of tabulated data, in Proceedings of the IEEE Conference of Electrical Performance of Electronic Packaging, 2003, pp. 295-298.

[35] D. Saraswat, R. Achar, and M. Nakhia, On passivity check and compensation of macromodels from tabulated data, in Proceedings of the 7th IEEE Workshop on Signal Propagation on Interconnects, Siena, Italy, 2003, pp. 25-28.

[36] C. Schröder and T. Stykel, Passivation of LTI Systems, Technical report 368, DFG Research Center Matheon, TU Berlin, Berlin, 2007.

[37] G. W. Stewart and J.-G. Sun, Matrix Perturbation Theory, Academic Press, New York, 1990.

[38] L. N. Trefethen and M. Embree, Spectra and Pseudospectra: The Behavior of Nonnormal Matrices and Operators, Princeton University Press, Princeton, NJ, 2005.

[39] V. A. Yakubovich and V. M. Starzhinskis, Linear Differential Equations with Periodic Coefficients, Vol. 1, John Wiley \& Sons, New York, Toronto, 1975.

Copyright ( by SIAM. Unauthorized reproduction of this article is prohibited. 